University of Zurich

Department of Economics

Working Paper Series

ISSN 1664-7041 (print)

ISSN 1664-705X (online)

Working Paper No. 299

\title{
Is Social Responsibility a Normal Good?
}

Björn Bartling, Vanessa Valero and Roberto A. Weber

September 2018 


\title{
Is Social Responsibility a Normal Good?
}

\author{
Björn Bartling Vanessa Valero Roberto A. Weber \\ University of Zurich
}

September 14, 2018*

\begin{abstract}
We investigate whether growth in consumer income causes an increased willingness to pay to mitigate negative externalities from consumption. Correlational field evidence suggests a positive relationship between income and social responsibility. To investigate a causal link, we conduct a laboratory market experiment in which firms and consumers can exchange products that differ in the degree to which they mitigate negative external impacts at the expense of higher production costs. Our treatments exogenously vary consumers' incomes. Our experimental results reveal that growth in consumer income causes an increase in the share of socially responsible consumption in the laboratory. Such a causal relationship is significant from a policy perspective, as it implies that some negative external impacts of consumption activity can be mitigated as societies experience economic growth.
\end{abstract}

JEL Classification: C92, D31, D62, M14

Keywords: Social responsibility, income growth, normal goods, laboratory experiment, market game

\footnotetext{
* Department of Economics, Blümlisalpstrasse 10, 8006 Zurich, Switzerland, email: bjoern.bartling@econ.uzh.ch, vanessa.valero@econ.uzh.ch, roberto.weber@econ.uzh.ch. We are grateful for support from the Swiss National Science Foundation (grant number 100018_165943) and for helpful comments from participants at several seminars, workshops and conferences.
} 


\section{Introduction}

Negative externalities resulting from market activity, such as environmental damage, create potentially serious social problems. Public authorities may often fail to correct for market failures, for instance, due to lobbying, regulatory capture or other limitations inherent to political processes. As a result, consumer social responsibility presents a potentially valuable complementary approach to preventing inefficiencies caused by market failures (Bénabou and Tirole, 2010). However, voluntarily mitigating externalities is costly for individual consumers, as the prices of socially responsible products are typically higher than prices of comparable products that create more external costs. Thus, understanding the factors that influence socially responsible market behavior by consumers - and that can, therefore, be employed to increase such behavior - is important for policy and welfare (e.g., Danz et al. 2012, Bartling et al. 2015, Kirchler et al. 2015, Pigors and Rockenbach 2016, Engelmann et al. 2018).

One potentially important factor in this regard is whether economic growth and thus increased income lead consumers to have greater willingness to pay in order to avoid negative externalities from their purchases. If this is the case, economic growth itself might play a critical role in the establishment of more socially responsible societies in which consumers voluntarily internalize their market impacts to a greater extent. As an example, if socially responsible consumption is, in fact, a normal good, then the substantial increases in household wealth in countries like China over the past several decades may yield an increased shift in consumer behavior toward more socially responsible products.

Consistent with this possibility, some scholars have previously argued for a positive relationship between income and socially responsible behavior. For instance, Shleifer (2004) hypothesizes that as "societies grow richer, their willingness to pay for ethical behavior [...] increases as well” (p. 418). Similarly, Bénabou and Tirole (2010) argue that "social responsibility is likely to be a normal good” (p. 1). However, we know of no empirical evidence that directly supports these conjectures by demonstrating such a causal positive relationship.

One way to investigate whether greater income produces more socially responsible behavior is to study relationships between consumer's expenditures and their income, both across countries and across time. In Figure 1, we combine cross-sectional country level data on GDP per capita and on Fair Trade expenditure as a share of consumer spending to provide correlational evidence on the relationship between consumer income and social 
responsibility. ${ }^{1}$ The figure reveals a positive relationship, which is consistent with the notion that social responsibility is a normal good. However, the field data do not establish a causal relationship, as countries can differ in dimensions that jointly determine GDP per capita and socially responsible consumption behavior. For example, the population in some countries might be more patient and forward-looking, leading to higher levels of capital accumulation and GDP and to a pronounced concern about long-term effects of economic activity on the environment. Second, consumers might perceive fair trade products to be different from conventional products not only in their social impact but also in their quality. The relationship in Figure 1 might thus reflect consumers switching to products of higher quality when their incomes increase, rather than increased concern with mitigating external harm.

Fig. 1: Share of consumer expenditure on fair trade products and GDP per capita in 2013

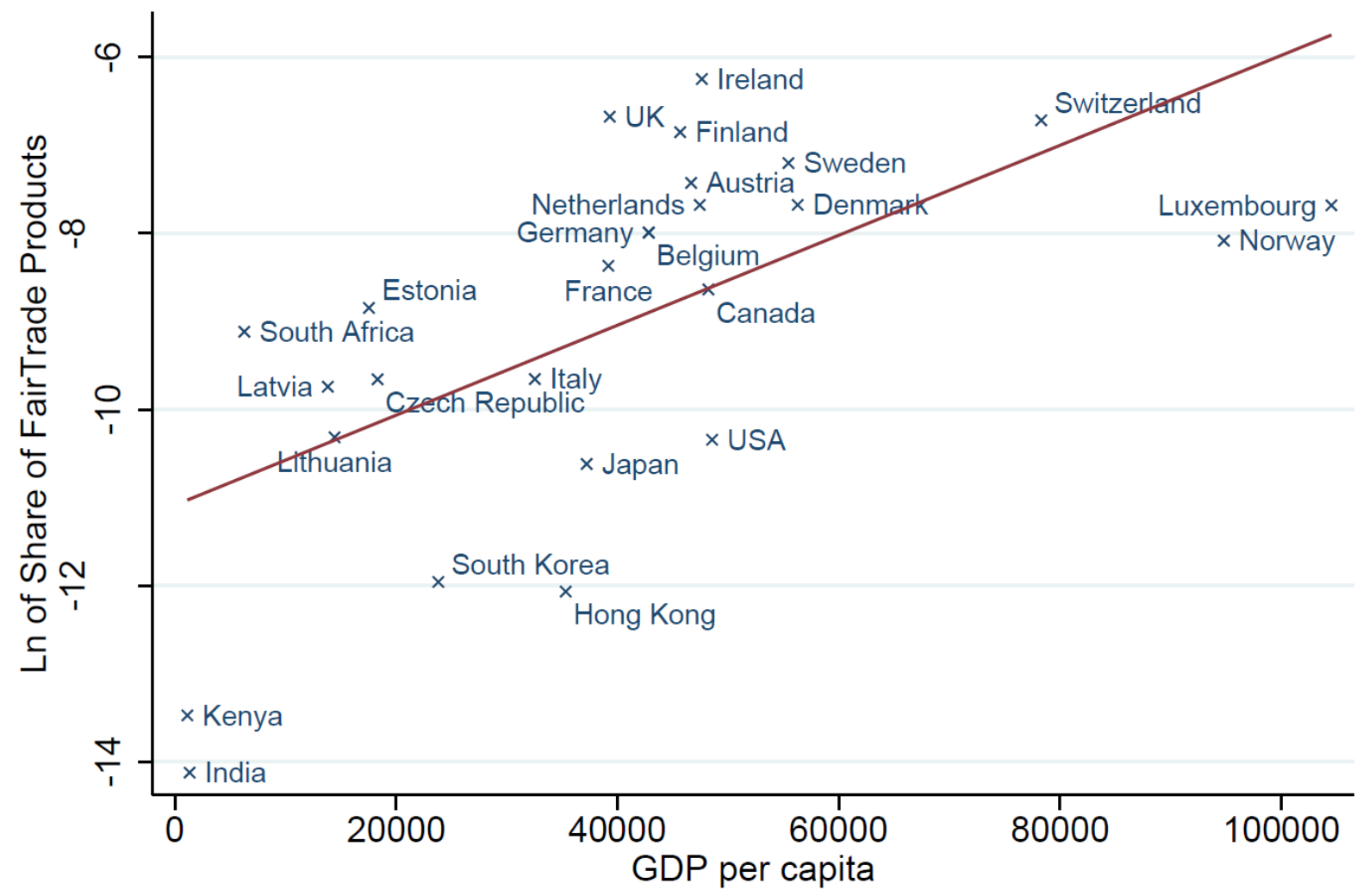

Notes: Own calculations based on data from the World Bank database and Fairtrade Labelling Organizations International (FLO), Annual Report 2013/14. GDP in 2013 US dollars.

\footnotetext{
${ }^{1}$ Figure 1 includes all countries for which we were able to obtain spending data on Fair Trade products for 2013. Appendix A reports the correlation, over time, between GDP per capita and the share of consumers' total expenditures spent on Fair Trade products in a sample of countries. We find a positive correlation between GDP and Fair Trade market share in 15 out of the 17 countries for which we have data on both measures from 2004 to 2013. The data set we use for this analysis is available at: https://www.dropbox.com/sh/ps13e5fey99sn6d/AADrJviPp4ujzWjhOqXUvBGpa?dl=0.
} 
Figure 1 highlights the challenges with attempting to demonstrate a clear causal connection between consumer income and socially responsible consumption. The necessary data require both exogenous variation in income and expenditures on products that differ only in the extent to which they produce varying degrees of social harm. In fact, such data is unlikely to exist in naturally occurring markets. Therefore, in this paper we investigate this relationship using a laboratory experiment, where it is possible to both vary earnings exogenously and to construct stylized versions of consumer products that vary only in their social impact. Of course, laboratory results naturally raise concerns about external validity, thus highlighting the complementary value of observing positive relationships between income and socially responsible consumption both in our experimental data and in Figure 1.

Subjects in our experiment play one of three roles: consumers, sellers or third parties. Sellers make production decisions involving prices and the degree of negative externalities produced by a product, with products that mitigate a larger degree of the negative externality costing more to produce. Consumers then observe product offers and select one from the available options. Any resulting negative externality from a consumer's purchase decision affects the passive third parties who serve no other function in the experiment. In our design, consumers have considerable market power, meaning that their preferences exert a driving force on market outcomes. Thus, we can measure social responsibility by the degree to which consumers' product purchases mitigate the potential losses imposed on third parties. Using this design, we study the impact of exogenously increasing consumers' incomes on their willingness to pay in order to avoid such negative social impact.

Our experiment yields several insights. Most importantly, we find that increasing consumers' incomes leads to higher levels of socially responsible market behavior, reflected in a greater willingness to purchase more expensive products that produce lower externalities. However, this effect is very modest for moderate income increases and is only large in magnitude and statistically significant when the income increases are substantial-e.g., corresponding in size, roughly, to the increase in GDP per capita between 2006 and 2017 in China.

Second, we also observe firms' reactions to increased consumer income. Interestingly, part of the increased willingness by consumers to purchase socially responsible products is captured by firms' increased markups for these types of products. This observation suggests a potential impediment to the mitigation of social harm through heightened consumer social responsibility brought about by income increases-some of the change in consumer behavior may not result in more socially responsible products, but rather in consumers paying more for 
such products. Firms may use the fact that high-income consumers are willing to pay for socially responsible products as an indication that they are less price sensitive, thereby allowing firms to obtain rents in serving such consumers.

Finally, our paper investigates two other aspects of income growth, and how they may influence whether individuals exhibit greater social responsibility as they become wealthier. First, we study how the distribution of income affects the relationship between income growth and social responsibility. Economic growth often leads to income gains that are concentrated in small subsets of a country's population (e.g., Alvaredo et al. 2013). We explore this issue with two treatments that hold constant the aggregate total increase in income, but vary whether this increase is equally distributed among all consumers in a market or concentrated as much larger increases for a small subset of the consumers. Second, we also investigate whether relative income comparisons matter, beyond absolute levels of income. Across treatments, we observe consumers who end up with the same levels of income, but are either in markets where all other consumers experience similar income increases or in a market where some other consumers have income shocks that are either larger or smaller. We find that social responsibility does not respond either to income inequality or to how much others' income changes_-only absolute income changes appear to influence social responsibility.

Our paper proceeds as follows. The next section briefly reviews some related work on how income and wealth influence prosocial and moral behavior. Section 3 then describes our experimental design, while Section 4 presents the results. Section 5 concludes.

\section{Related Literature}

Our purpose is to test the hypothesis that higher income leads consumers to exhibit greater concern for social responsibility in their purchasing behavior. As we note above, prominent economists have conjectured the existence of such a relationship (Shleifer 2004, Bénabou and Tirole 2010), but we are not aware of clean causal evidence in support of this relationship.

Consistent with our observation in Figure 1, other correlational evidence supports the idea that pro-social behavior is higher among individuals with greater income. This is evident, for instance, in studies that investigate the relationship between income and charitable giving, which regularly observe that wealthier individuals donate more in absolute terms, though the question of whether they give more in relative terms remains inconclusive (e.g., Andreoni 2006, Vesterlund 2006, Andreoni and Payne 2013). As with our analysis in Figure 1, however, it is hard to rule out that other factors, such as differential tax treatment of large and small donations, may be at least partly responsible for these relationships. 
A recent study by Andreoni et al. (2017) investigates whether rich or poor households are differentially likely to return an envelope with money that was purportedly mistakenly delivered to their address. They identify 180 rich and 180 poor households in the Netherlands using register data and send them letters containing either 5 Euro or 20 Euro intended for another recipient. The results reveal that over twice as many rich households (81 percent) return the envelopes as poor households (38 percent). This suggests that the rich households are more concerned with returning the money to which they are not entitled. Analysis that includes controls for monthly variation in liquidity leads the authors to conclude that the differences can be attributed to the rich and poor's facing different financial constraints. This highlights similar issues to those in Figure 1-a household's status as either rich or poor is not exogenous and other covarying factors may influence the differential pro-sociality in returning the envelopes between the two groups.

Other, also largely correlational, evidence suggesting a negative relationship between wealth and morality is provided by social psychologists. Specifically, in a series of studies, Piff et al. (2010, 2012) examine whether upper-class individuals are more likely to exhibit unethical behavior (e.g. break the law, lie to get ahead, and cheat in games). Their findings confirm their hypothesis that having more money makes people care less about others and feel more entitled to put their own interests first. However, in their studies, social class is typically not varied exogenously, meaning that individuals with high and low social class differ in other dimensions than income (such as level of education and occupational status). In addition, many of the outcome measures involve hypothetical choices.

None of the above studies implements exogenous variation in income. Perhaps the most relevant evidence in this regard comes from laboratory experiments on the impact of income in dictator games. Several studies conduct dictator games with varying stake sizes (e.g., Forsythe, et al. 1994, Cherry et al. 2002, Carpenter et al. 2005). Engel (2011) conducts a metaanalysis of dictator games and finds that the absolute amounts shared by dictators increase substantially when stake sizes increase-a 100-percent increase in a dictator's endowment produces an increase slightly below 100 percent in the amount transferred. Thus, prosociality, as measured by the absolute size of transfers to a recipient, increases with income with an elasticity close to 1 . However, there are many reasons to be skeptical that these degrees of income elasticity are likely to apply to socially responsible behavior by consumers in a market context. For starters, dictator games possess a clear norm to share 50 percent of the endowment (e.g., Andreoni and Bernheim 2009, Krupka and Weber 2013). Such strong norms are consistent with elasticities close to 1 , but limit generalization to contexts where such strong 
50/50 norms are not applicable, including product purchases by consumers. In addition, prior evidence (e.g., Bartling et al. 2015) suggests that the degree of social concern exhibited by individuals can differ substantially between dictator games and market contexts.

To our knowledge, only one other paper experimentally investigates whether social responsibility is a normal good. Friedrichsen (2017) studies markets with one consumer, two firms and one worker. The two firms set wages for the worker and the consumer then decides how much to purchase from each firm. Consumers in each session were randomized to have either a low or high income. Friedrichsen defines social responsibility as a consumer buying a larger quantity from a firm that sets a strictly higher price but also pays a strictly higher wage than from a low-wage, low-price competitor. In total, 13.8 percent of consumer choices indicate a preference for the socially responsible product. Interestingly, richer consumers are less likely to favor the socially responsible product $(10.2 \%)$ than are poor consumers $(17.7 \%)$. While seeming to support Piff et al.'s $(2010,2012)$ contention that higher income produces diminished concern for social impact, a few design features suggest the need for further investigation. First, the numbers of poor (18) and rich (19) consumers in the study are relatively low. Second, average payoffs for workers (29.06 points per period $\approx 0.29$ Euro) are actually higher than average payoff for firms (26.42). Hence, a socially responsible consumer concerned with equality may actually want to help firms at the expense of workers, buying more from the low-cost, low-wage firm. Nevertheless, Friedrichsen's (2017) results raise the interesting possibility that, contrary to our hypothesis, social responsibility may be an inferior good.

\section{Experimental Design}

In our experimental markets, firms and consumers can exchange products that differ in their social impact, with products that impose a smaller externality on third parties also costing more to produce. The experiment comprises 30 periods, with the first 10 periods ("Part I") corresponding to a baseline design that is identical across all treatments. To study the effect of an exogenous income increase on socially responsible market behavior, our treatments introduce varying shocks to consumers’ incomes in periods 11-30 (“Part II”).

\subsection{Baseline Market Game}

Each of our experimental markets comprises 18 participants: six firms, six consumers and six passive third parties. The participants and roles in a market are fixed across all 30 periods of the experiment. Every market begins with 10 periods of the baseline version of our design, 
where every firm, consumer and third party receive an income of 100 points. Firms and consumers can earn additional points by trading products, with products of varying types having different production costs and varying impacts on third parties.

At the beginning of a market period, every firm selects a type of product to offer and a price. A product's type corresponds to the total loss it imposes on third parties when purchased, which is between 0 and 60, and a corresponding production cost. The total loss is divided equally and imposed on each of the six third parties. Table 1 provides an overview of the 11 possible product types that a firm can offer, the associated combined and individual losses for third parties and the respective production costs. A key feature of our design is that products that impose a smaller externality on third parties also cost more to produce. A decrease in the combined loss to third parties of six-and, therefore, a decrease of one for the loss imposed on each third party-increases the production cost by one.

Table 1: Product types, losses for third parties, and production costs

\begin{tabular}{lcccccccccccc}
\hline & \multicolumn{11}{c}{ Product types } \\
\hline Combined loss & 0 & 6 & 12 & 18 & 24 & 30 & 36 & 42 & 48 & 54 & 60 \\
Individual loss & 0 & 1 & 2 & 3 & 4 & 5 & 6 & 7 & 8 & 9 & 10 \\
\hline Production cost & 30 & 29 & 28 & 27 & 26 & 25 & 24 & 23 & 22 & 21 & 20 \\
\hline \hline
\end{tabular}

At the same time as they select product types, firms also determine prices for their product offers. ${ }^{2}$ Products are worth 70 to consumers, independently of the degree of externality they impose on third parties. ${ }^{3}$ Firms are required to set prices between the production cost of the selected product type and 70.

Firm's offers are conveyed to consumers in a posted-offer market. Offers are displayed in a random order. After firms make their decisions, consumers see the prices and types of the six products offered in that period. Each consumer can buy one product but can also decide not to buy any product. A decision not to buy a product yields no profits for either the consumer or any firm, but also means no losses for third parties. There is no capacity constraint on the supply side; that is, each firm can serve the entire market and sell up to six units of the offered product.

\footnotetext{
${ }^{2}$ The interfaces for firms' and consumers' decisions are provided in Appendix B.

${ }^{3}$ The fact that we hold constant the characteristics of a product other than its social impact is a valuable element of the control afforded by a laboratory environment.
} 
The third parties are passive participants and do not make any decisions. However, their payoffs in a period are impacted by the types of products exchanged in the market. Specifically, each third party experiences a loss between 0 (whenever all consumers either only buy products that produce no loss or do not buy products at all) to 60 (whenever all consumers buy the product that produces the maximum possible loss).

Players' final payoffs, in points, in a given period are thus as follows:

$$
\begin{aligned}
& \pi_{\text {Consumer }}=100+(\text { price }- \text { cost }) \cdot \text { quantity purchased }, \\
& \pi_{\text {Firm }}=100+(\text { price }- \text { cost }) \cdot \text { quantity sold }, \\
& \pi_{\text {Third Party }}=100-\text { sum of losses from products exchanged, }
\end{aligned}
$$

where the quantity purchased by a consumer is either 0 or 1 , the quantity sold for a firm can be any integer from 0 to 6 and the sum of losses for a third party ranges from 0 to 60 . At the end of every period, players observe their own payoff. In addition, firms observe the offers made by all firms, how many products they sold, their payments and the impact of the products they sold on Participants C. Consumers observe the effect of their purchasing decision on the payments of the six Participants C. ${ }^{4}$ Individual subjects are not identified to one another-i.e., there are no identification numbers associated with feedback—and therefore cannot track each others' actions across periods.

This baseline design is very similar to the one in Bartling et al. (2015), but there are several important differences that are intended to mimic more natural consumer product market contexts. First, rather than a binary product type, our design allows for varying degrees of social impact, with the productions costs proportional to the degree of externality mitigation. Second, rather than having a product externality that has a large impact on only one third party, each product has a smaller impact on a larger number of individuals (see Bartling et al. (in press) for a comparison of these two types of external impacts). Finally, we introduce much harsher competition on the supply side, by allowing each firm unlimited production capacity.

\subsection{Treatments}

As we note above, consumers' initial per-period income is 100 in the first 10 periods in all our treatments. To study the causal effect of consumers' income levels on socially responsible market behavior, our design exogenously varies the size of consumers' initial per-period

\footnotetext{
${ }^{4}$ The screenshots of the feedback provided to participants can be found in Appendix C.
} 
income in the remaining 20 periods. We implement this design—rather than one in which consumers have varying income levels from the outset-for two reasons. First, providing consumers across treatments with the same initial per-period income in Part I gives us a baseline measure of social responsibility at the consumer and market level. Second, increasing income in Part II relative to Part I implements differences in income growth, which more directly relates to our research question of whether we can expect an increase in socially responsible market behavior as societies become richer. Hence, much of our analysis will consist of difference-in-difference estimates of the responses of consumers in the treatment conditions who receive positive income shocks, relative to the comparable behavior in a control condition that implements the baseline design for all 30 periods. Our first condition is thus a Baseline control that implements the same per-period income of 100 in Part II, as in Part I.

Our three remaining treatments implement positive income shocks for consumers in all periods of Part II. At the end of Part I subjects receive new instructions that explain the change in initial income for consumers and that, otherwise, all procedures remain the same. For comparability, subjects also receive new instructions in the Baseline.

Table 2 provides an overview of our treatments. In a Medium condition consumers each receive 200 in income at the start of a period throughout Part II, while in a High condition they each receive 400. ${ }^{5}$ Finally, in an Unequal condition two consumers in each market receive 400, while the remaining four consumers continue to each receive 100. Note that this implements an average increase of 200, as in the Medium condition, but that these gains are concentrated on a small subset of consumers.

Thus, by comparing, in Part II, the types of products purchased in all three conditions receiving a positive income shock with the Baseline, we can identify whether social responsibility is a normal good. Furthermore, a comparison of Unequal and Medium allows us to study the extent to which an aggregate change is influenced by the distribution of income. Finally, we can also compare the behavior of consumers in Unequal, who receive income of either 100 or 400, to the behavior of those consumers in the Baseline and High who have the same initial per-period income level. Note that consumers in the Baseline and those consumers earning 100 in the Unequal condition receive the same absolute income of 100, but differ in their income relative to other consumers in their market. Similarly, consumers who receive 400

\footnotetext{
${ }^{5}$ These increases_-doubling and quadrupling of income-roughly correspond, respectively, to the changes in GDP per capita (measured in 2018 US dollars) in China between $2009(\$ 3,838)$ and $2017(\$ 8,827)$ and 2006 $(\$ 2,099)$ and 2017. Data from the World Bank: https://data.worldbank.org/indicator/NY.GDP.PCAP.CD?locations=CN.
} 
in the Unequal condition receive the same absolute income as those in the High condition, but have a high relative income in comparison to other consumers in their market.

Table 2: Treatment overview

\begin{tabular}{|c|c|c|c|c|}
\hline \multirow[b]{2}{*}{ Treatment } & \multicolumn{2}{|c|}{ Consumers' per-period income } & \multirow{2}{*}{$\begin{array}{l}\text { Number of } \\
\text { markets } \\
\text { (participants) }\end{array}$} & \multirow{2}{*}{$\begin{array}{l}\text { Number of consumers in } \\
\text { a market (total number of } \\
\text { consumers) with a given } \\
\text { initial income }\end{array}$} \\
\hline & $\begin{array}{c}\text { Part I } \\
\text { (periods } 1 \text { to 10) }\end{array}$ & $\begin{array}{c}\text { Part II } \\
\text { (periods } 11 \text { to 30) }\end{array}$ & & \\
\hline Baseline & 100 & 100 & 10 (180) & $6(60)$ \\
\hline Medium & 100 & 200 & $10(180)$ & $6(60)$ \\
\hline High & 100 & 400 & $6(108)$ & $6(36)$ \\
\hline \multirow{2}{*}{ Unequal } & 100 & 100 & \multirow{2}{*}{16 (288) } & $4(64)$ \\
\hline & 100 & 400 & & $2(32)$ \\
\hline
\end{tabular}

\subsection{Procedural Details}

As shown in Table 2, we conducted 10 markets in the Baseline, 10 markets in the Medium condition, 6 markets in the High condition and 16 markets in the Unequal condition. Our objective was to obtain 60 consumers in each treatment, but the substantial cost of the High condition limited the number of sessions we conducted. ${ }^{6}$ We implemented a between-subjects design. In total, 756 subjects participated in our study, in 42 independent markets.

Before entering the lab, each subject randomly drew a card that specified at which computer terminal to sit, which also determined the subject's role. Subjects received written instructions, including comprehension questions that had to be answered correctly before the market began. An audio file with a summary of the instructions was played aloud with the intent to establish common information about actions, payoffs and procedures. Subjects initially learned only the details of Part I, but were informed that Part II would follow and would consist of the same kind of market activity. We provided information to the subjects about the details of Part II only after the conclusion of Part I. All our treatments are thus identical in Part I. We introduced an explicit market context in the instructions. We described the two different types of market participants as "firms" and "consumers" and referred to their actions as "trading” different "types of products" at offered "prices.” The third parties were

\footnotetext{
${ }^{6}$ To determine the power of our design to detect a significant difference at the $5 \%$ level, we used the effect size in Forsythe et al. (1994), who increase stakes from $\$ 5$ to $\$ 10$ in a dictator game. The power analysis indicates that the probability of detecting a two-sided effect between any two treatments in a Wilcoxon rank-sum test is about $90 \%$ if we use consumers as unit of observation and have a minimum of 32 observations per treatment.
} 
simply described as "Players C." We told the subjects in neutral language that a type of product "refers to the individual loss that a product imposes on each Participant C.” At the conclusion of Part I, subjects received new instructions stating that the market procedures would be the same in Part II and specifying each type of participant's income, including noting any relevant changes. The original English instructions for all treatments are in Appendix $\mathrm{H}$.

We conducted the experiments using the software z-Tree (Fischbacher 2007) and recruited subjects using the software h-Root (Bock et al. 2014). Subjects were mainly students from the University of Zurich and the Swiss Federal Institute of Technology in Zurich. At the end of a session, participants completed a questionnaire on socio-economic background, political views, real-world purchasing behavior and charitable giving. The list of all elicited variables, their description and summary statistics are in Appendix D. All sessions took place at the computer laboratory of the Department of Economics at the University of Zurich. Sessions lasted about two hours.

At the end of the experiment, one period was selected at random to count for payment. Payoffs from the experiment, denominated in points, were converted to Swiss francs at the rate of 3 points per CHF 1 (Swiss francs and US dollars were roughly at parity at the time of the study). On average, subjects earned about CHF 61, which includes a show-up fee of CHF 15.

\section{Results}

We define our measure of socially responsible behavior as the percentage of the total potential loss that is mitigated through consumers' product purchases. Hence, if market participants exchange only the most harmful types of products, which impose a loss of 60 on third parties, they are not mitigating any loss and the social responsibility measure is 0 percent. On the other hand, if they mitigate the entire externality-either by incurring the maximal production cost or by not trading - then this measure is 100 percent.

Figure 2 shows the time paths of this measure of social responsibility in all four treatments. To smooth variation, we pool the data into two-period blocks. A first observation is that, in Part I, our measure of social responsibility is similar in all treatments, at approximately 33 percent. $^{7}$ The treatments do not differ significantly, confirming successful randomization of subjects into treatments. ${ }^{8}$

\footnotetext{
7 The percentage of the total potential loss that is mitigated through consumers' product purchases for each treatment and at each part can be found in Table E1 in Appendix E.

${ }^{8}$ None of the pairwise treatment comparisons for Part I is statistically significant in two-sided Wilcoxon ranksum tests, neither at the consumer nor at the market level (see Table E2 in Appendix E).
} 
Figure 2: Average social responsibility across treatments

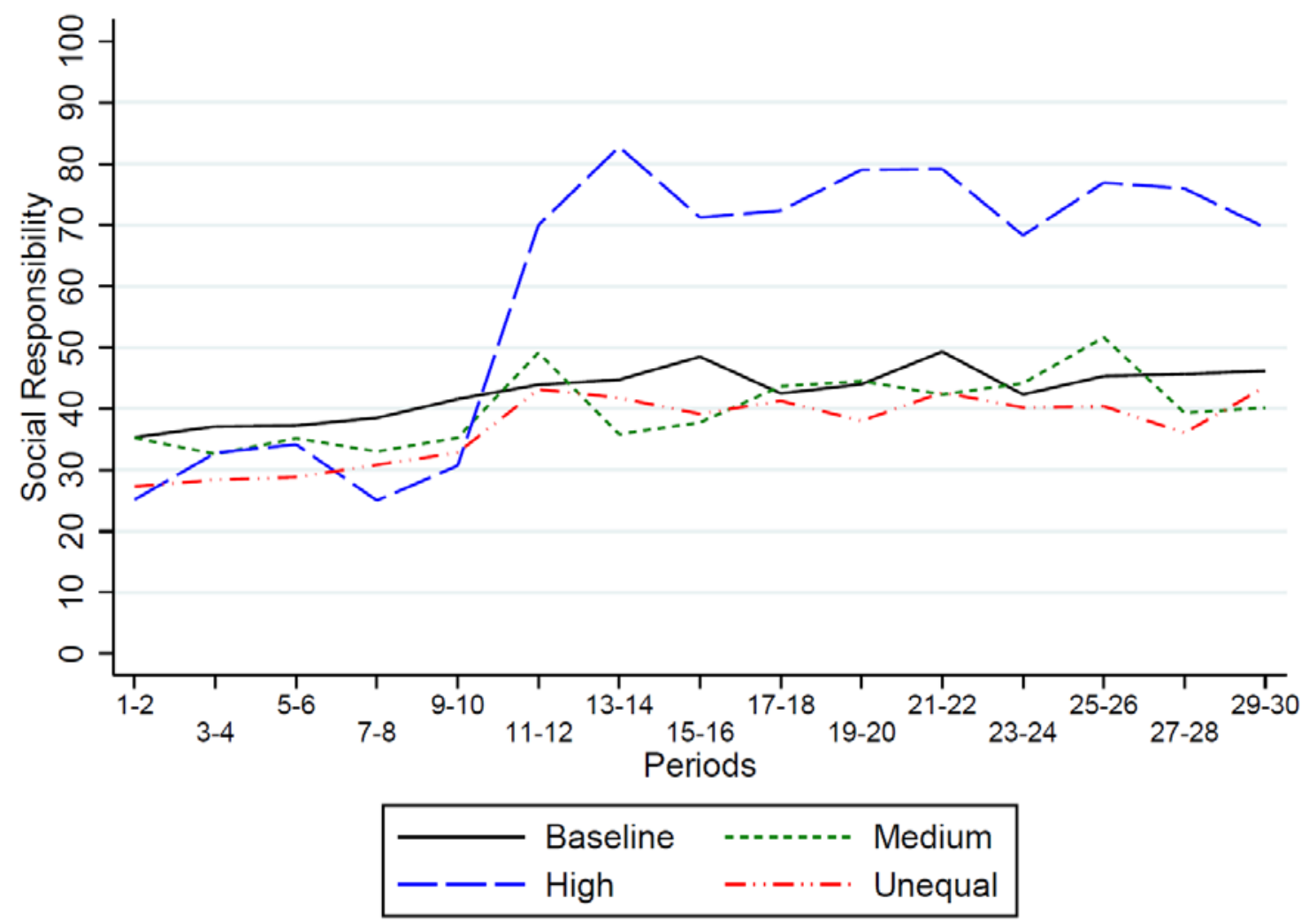

\subsection{Income Growth and Social Responsibility}

Our primary focus is on the impact of increasing consumers' incomes in Part II on social responsibility. We first focus on comparisons between the Baseline, Medium and High conditions. Market behavior in Part II in the Baseline allows for clean comparisons that control for any behavioral changes that might develop with market experience over the course of the experiment.

Figure 2 reveals that social responsibility increases only very slightly in Part II in Medium. Indeed, Baseline and Medium are not statistically significantly different in Part II (two-sided Wilcoxon rank-sum tests at the consumer (market) level, $\mathrm{p}=0.83$ (0.65)). However, Figure 2 shows that social responsibility increases substantially in Part II in High. The difference between Baseline and High is statistically significant in Part II (two-sided Wilcoxon rank-sum test, at the consumer (market) level, $\mathrm{p}=0.00(0.04)$ ), as is the difference between Medium and High ( $\mathrm{p}=0.00(0.01))$. 
Table 3: GLS (random-effects) regression of social responsibility measure

\begin{tabular}{lccc}
\hline \hline & $(1)$ & $(2)$ & $(3)$ \\
\hline Medium & -3.700 & -3.467 & -0.023 \\
High & $(9.463)$ & $(10.093)$ & $(9.591)$ \\
Unequal & -8.394 & -8.582 & -7.667 \\
& $(13.079)$ & $(13.423)$ & $(12.673)$ \\
Part II & -8.325 & -9.643 & -7.712 \\
& $(8.853)$ & $(9.600)$ & $(9.007)$ \\
Part II x Medium & $7.300^{*}$ & $7.473^{*}$ & $7.473^{*}$ \\
& $(3.843)$ & $(4.123)$ & $(4.124)$ \\
Part II $x$ High & 1.308 & 2.334 & 2.334 \\
Part II $x$ Unequal & $(6.340)$ & $(6.460)$ & $(6.463)$ \\
Disposable income (log) & $37.686 * * *$ & $36.693^{* * *}$ & $36.693^{* * *}$ \\
& $(8.396)$ & $(9.625)$ & $(9.629)$ \\
Responsible consumption & 3.710 & 3.581 & 3.581 \\
Donation to charity & $(4.933)$ & $(5.231)$ & $(5.233)$ \\
Political orientation (right) & & & $-7.203^{* * *}$ \\
Constant & & & $(2.775)$ \\
& & & $12.212^{* * *}$ \\
Controls for age and gender & & & $(4.121)$ \\
Observations & & & $0.017 * * *$ \\
Number of subjects & & & $(0.004)$ \\
\hline \hline
\end{tabular}

Notes: Medium, High and Unequal are binary variables taking on value 1 in the respective treatments and 0 otherwise. Part II is a binary variable taking on value 1 for data from period 11 to 30 and 0 otherwise. Baseline and Part I serve as omitted categories. Disposable income is the natural logarithm of subjects' self-reported disposable monthly income. Responsible consumption is a binary variable taking on value 1 if subjects "often" or "always" buy socially responsible products (and 0 if they "sometimes" or "never" buy them). Donation to charity are self-reported donations to charitable causes (in CHF) per year. Political orientation captures subjects' political preferences on a nine-point scale, ranging from "very left-wing" to "very right-wing." We have only 239 subjects in models (2) and (3) because 13 subjects did not declare either their disposable income or their responsible consumption behavior. Standard errors (in parentheses) are clustered at the market level. * significant at $10 \%,{ }^{* *}$ significant at $5 \%,{ }^{* * *}$ significant at $1 \%$.

Table 3 presents regressions that complement these observations. The table reports coefficient estimates of random-effects regressions using a consumer's purchasing decision in a period as the unit of observation. The dependent variable is our measure of social responsibility (percent loss mitigated). To accommodate market heterogeneity, we use a 
generalized least-squares (GLS) panel structure with random effects at the consumer level. We employ a standard differences-in-differences structure for panel data to examine treatment effects in Part II conditional on behavior in Part I. In Model (1) we include binary treatment variables, Medium, High and Unequal, taking on value 1 in the respective treatments and 0 otherwise, a binary variable Part II, taking on value 1 for data from periods 11 to 30 and 0 otherwise and interactions of the treatment variables with Part II. Baseline and Part I serve as omitted categories.

Model (2) repeats this analysis using only consumers for whom we have complete information on individual characteristics collected in the post-experimental questionnaire, which has little substantive effect on the results. Model (3) introduces these individual characteristics as explanatory variables. Disposable income refers to (the natural logarithm of) subjects' self-reported disposable monthly income (i.e., after housing costs). Responsible consumption indicates how often a subject buys socially responsible products; subjects could select “always,” “often,” "sometimes,” or “never;” we collapse this into two roughly equallysized categories: sometimes/never (0) and often/always (1). Donation to charity corresponds to the amount donated (in CHF) per year to charitable causes. Finally, Political orientation captures subjects' political preferences, ranging from 1 if they consider themselves very much on the left of the political spectrum to 9 if they consider themselves on the right extreme. We also include, in addition, controls for age and gender.

Across all models, the positive coefficients for Part II $x$ Medium indicate slightly increased concern for social impact when consumers' initial income increases from 100 to 200, but the impact is small and not statistically significant. We observe similarly small and positive, but statistically insignificant, effects for the Unequal condition in Part II. However, the coefficient for Part II $x$ High is positive, much larger in magnitude and significant at the one percent level. This confirms a large impact on socially responsible consumption when income increases substantially.

We can also directly estimate the effects of income levels on socially responsible consumption. Table 4 presents results from regressions of our measure of social responsibility on a consumer's income in a period, using data from all treatments. The identification of income effects in this regression comes from the variation between subjects and across time in the size of the per-period income. Both models use the level of socially responsible consumption as the dependent variable and the level of income as an explanatory variable, revealing a positive and statistically significant relationship. An increase of 100 units of income leads to an increase of roughly 12 in our measure of social responsibility. The positive and 
statistically significant coefficients for Income are consistent with the interpretation that socially responsible consumption is a normal good.

Table 4: GLS (random-effects) regression of social responsibility

\begin{tabular}{|c|c|c|}
\hline & (1) & (2) \\
\hline Income & $\begin{array}{c}0.121 * * * \\
(0.016)\end{array}$ & $\begin{array}{c}0.115^{* * *} \\
(0.017)\end{array}$ \\
\hline Period & & $\begin{array}{c}0.114 \\
(0.098)\end{array}$ \\
\hline Constant & $\begin{array}{c}21.725^{* * *} \\
(4.096)\end{array}$ & $\begin{array}{c}21.067 * * * \\
(4.026)\end{array}$ \\
\hline Observations & 7,560 & 7,560 \\
\hline Number of subjects & 252 & 252 \\
\hline
\end{tabular}

The coefficient estimates in Models (1) and (2) of Table 4 also allow us to derive elasticities for the responses of social responsibility to increases in income. ${ }^{9}$ This yields elasticity estimates of approximately $0.55 .{ }^{10}$ Of course, these elasticity estimates from behavior in laboratory experiments should not be interpreted as likely to be particularly informative about comparable real-world income elasticities for the many different ways in which consumers can exhibit social responsibility in response to changing income. However, in the context of our experiment, they indicate a moderate level of responsiveness.

Returning to Table 3, Model (3) also yields some interesting insights regarding factors affecting socially responsible consumption. First, the coefficient on Responsible consumption is positive and statistically significant, revealing that those subjects who report more frequently consuming socially responsible products outside the laboratory also act more socially responsibly in our experimental markets. Second, the coefficient on Donation to charity is positive and highly significant, suggesting that subjects who report giving more to charity act more socially responsibly in our markets (see Sutter et al., 2016, for a comparable finding).

\footnotetext{
${ }^{9}$ Specifically, let social responsibility (the percent loss mitigated) be $S R^{0}$ and $S R^{1}$ before and after income changes, respectively, and let $\Delta S R=S R^{1}-S R^{0}$. Let $Y^{0}$ and $Y^{1}$ denote the corresponding per-period income levels and let $\Delta Y=Y^{1}-Y^{0}$. We calculate income elasticities as $\eta=\Delta S R / \Delta Y \cdot Y^{0} / S R^{0}$.

${ }^{10}$ In Table 3, using the estimates in Model (1), this yields estimated income elasticities in the range of 0.25 (in treatment Medium) to 0.51 (in treatment High).
} 
Third, the coefficient for Political orientation is negative and marginally statistically significant, suggesting that subjects who classify themselves toward the right of the political spectrum are less likely to exhibit socially responsible market behavior (see Sutter et al., 2016, and Cappelen et al., 2015, for comparable findings). Most interestingly for our main research question, the coefficient on Disposable income is negative and statistically significant, indicating that subjects with higher self-reported income levels outside the laboratory act less socially responsibly in our experimental markets. While our data are based on a student sample and might thus be of limited significance when it comes to interpreting variation in income levels outside the laboratory, this unexpected observation is interesting in light of work demonstrating negative relationships between income and social responsibility (e.g., Piff et al. 2010, 2012). It raises the possibility that wealthier individuals may be less pro-social than ones with lower incomes-perhaps due to many possible factors including selection into higher earnings - while the effects of our treatments suggest that the effects of exogenous income increases are positive. ${ }^{11}$

\subsection{Relative Income and Social Responsibility}

We next more closely study the Unequal condition, to investigate whether income inequality affects the relationship between income and social responsibility. Figure 2 shows that, on aggregate, the degree of socially responsible consumption in the Unequal condition is very similar to that in the Medium condition, both of which have the same aggregate income increases. Indeed, the measures of social responsibility in Unequal and Medium are not significantly different in Part II (two-sided Wilcoxon rank-sum test, at the consumer (market) level, $\mathrm{p}=0.58$ (0.71)). This is also reflected in the coefficient estimates for Unequal in Table 3, which show small positive, but statistically insignificant, estimates that are similar in magnitude to those for the Medium condition. ${ }^{12}$

\footnotetext{
${ }^{11}$ Table E3 in Appendix E reports further regression specifications, where we use alternative self-reported measures of income, responsible consumption behavior and political views. The results are substantively similar in these regression specifications. One observation is that the coefficient on Family income ("Approximately, what was the highest total gross income obtained by your parents in any past year?") is positive and statistically significant at the 10 percent level. This is again in line with the correlational evidence discussed at the beginning of the paper. The inconsistency between Family income and Disposable income suggests that any interpretation of these self-reported measures must be done very cautiously.

${ }^{12}$ A post-estimation test of equality of the coefficients for Part II $x$ Unequal and Part II x Medium in Model (1) fails to reject equality $(\mathrm{p}=0.68)$.
} 
Figure 3: Average social responsibility in treatment Unequal by income class

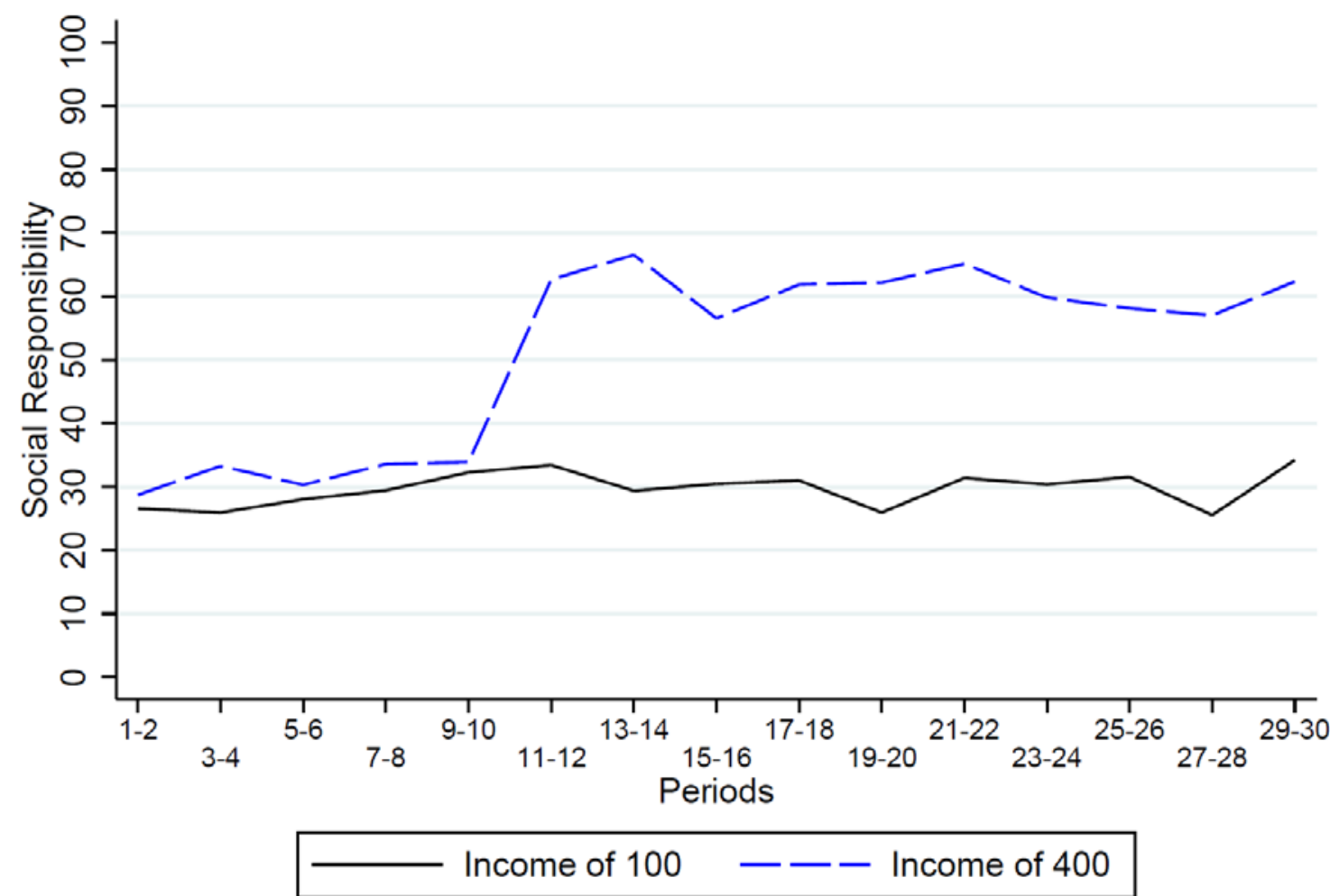

While the aggregate response to a positive income shock is small in the Unequal condition, we observe larger effects when we consider only those consumers whose income increased. Figure 3 shows that consumers whose initial per-period income increases from 100 in Part I to 400 in Part II in Unequal demonstrate considerably higher levels of social responsibility, while those consumers whose income remains at 100 demonstrate little change in their behavior. This observation is supported by the regression analysis reported in Model (1) in Table 5. This regression includes only observations from the Unequal condition to study how social responsibility is affected by the income shock experienced by two out of the six consumers in each market. Income of 400 is a binary variable taking on value 1 in case a consumer receives an initial per-period income of 400 in Part II and 0 otherwise. We find that the coefficient for Part II $x$ Income of 400 is positive and statistically significant, indicating that consumers whose initial income increases from 100 to 400 exhibit a substantially higher level of socially responsible behavior than consumers whose initial income stays constant at 100. This finding supports our earlier analysis comparing the Baseline and High conditions, finding that a substantial income increase (from 100 to 400) fosters increased socially responsible market behavior. However, in the case where there are only two consumers with the large income increase, the effect of their increased social responsibility is not sufficient to produce a large impact on aggregate behavior. 
Table 5: GLS (random-effects) regression of social responsibility

\begin{tabular}{lccc}
\hline \hline & $(1)$ & $(2)$ & $(3)$ \\
\hline Income of 400 & 3.516 & & \\
Unequal & $(7.004)$ & -9.497 & 2.413 \\
& & $(8.935)$ & $(12.408)$ \\
Part II & 1.883 & $7.300^{*}$ & $44.986^{* * *}$ \\
& $(2.124)$ & $(3.872)$ & $(7.551)$ \\
Part II x Income of 400 & $27.383^{* * *}$ & & \\
& $(4.931)$ & & -15.720 \\
Part II x Unequal & & -5.417 & $(9.666)$ \\
& & $(4.403)$ & $29.556^{* * *}$ \\
Constant & $28.453^{* * *}$ & $37.950^{* * *}$ & $(10.421)$ \\
& $(3.780)$ & $(8.118)$ & 2,040 \\
Observations & 2,880 & 3,720 & 68 \\
Number of subjects & 96 & 124 & \\
\hline \hline
\end{tabular}

Notes: Model (1) considers only observations from the Unequal treatment. Model (2) considers only observations from consumers who receive an income of 100 in Part II (Unequal and Baseline). Model (3) considers only observations from consumers who receive an income of 400 in Part II (Unequal and High). Income of 400 is a binary variable taking on value 1 in case a consumer receives an income of 400 in Part II and 0 otherwise. Unequal is a binary variable taking on value 1 in case of treatment Unequal and 0 otherwise. Part II is a binary variable taking on value 1 for observations from Part II and 0 otherwise. Standard errors (in parentheses) are clustered at the market level. * significant at $10 \%$, ** significant at $5 \%$, *** significant at $1 \%$.

The Unequal condition also allows us to investigate how relative income comparisons influence socially responsible market behavior. First, recall that the behavior of consumers who receive 100 in Part II in the Baseline, where all consumers receive 100, changes little from their behavior in Part I (see Figure 2 and Table 3). Our design allows us to compare the behavior of these consumers to the behavior of subjects who receive 100 in the Unequal condition, where some other consumers receive 400. Even though both sets of consumers experience no income changes, the latter may react to the presence of income changes among others in their market. However, Figure 3 reveals that the behavior of these consumers changes very little from Part I to Part II. This is confirmed in Model (2) in Table 4, which uses only observations from subjects who received income of 100 in Part II in the Baseline and Unequal conditions. We find that the interaction term Part II x Unequal is small, negative and statistically insignificant. Second, we can also compare socially responsible behavior in Part II by consumers who receive 400 in the High condition, where all consumers receive similar income shocks, to the behavior of the subset of consumers who receive 400 in the Unequal condition. Model (3) in Table 4 considers only observations from these two classes of subjects. The interaction term Part II $x$ Unequal is 
negative and sizable in magnitude, suggesting that the response to a positive income shock is smaller when other consumers simultaneously experience smaller shocks. However, the coefficient is not statistically significantly different from zero. Based on these results, we conclude that relative income comparisons do not appear to have large and robust effects on consumers' social responsibility.

\subsection{Prices and Markups}

Thus far, we have focused on the composition of product types-in particular, the extent to which traded products impose externalities on third parties-as our primary measure of consumer social responsibility, and how it is affected by increases in income. We next consider whether income changes also result in changes in the prices paid by consumers. Recall that all product types have the same value to consumers in material terms, meaning that a greater willingness to pay for more socially responsible products likely reflects an increased concern for mitigating negative impacts.

Figure 4: Average price paid

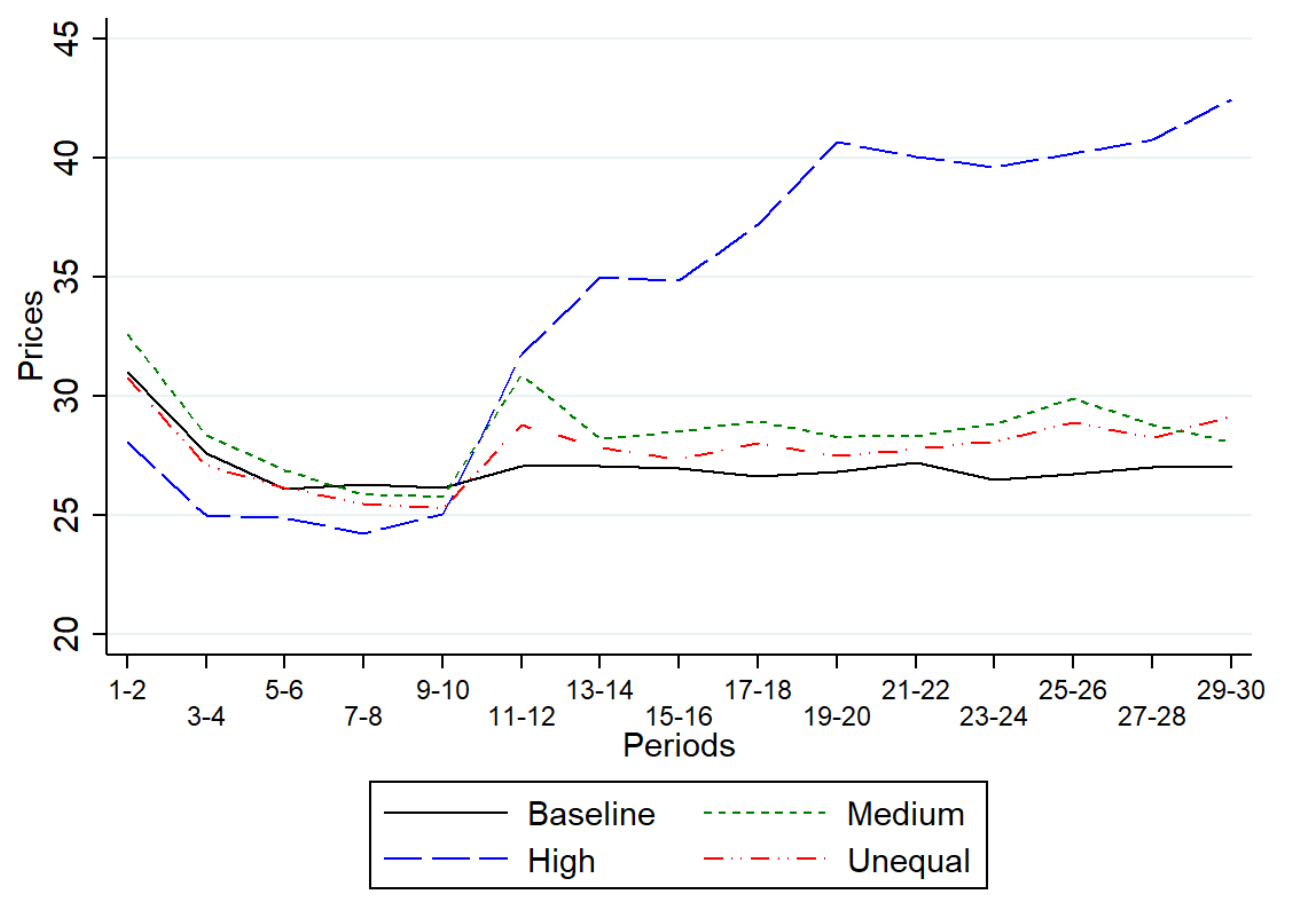

Notes: The figure shows average prices for all completed transactions.

We first report average prices paid by consumers, pooling the different types of products bought. Figure 4 shows the time path of the average prices paid in each treatment. On average, consumers pay a price of 26.9 in Part II in Baseline, 28.9 in Medium, 38.3 in High, 
and 28.2 in Unequal. ${ }^{13}$ Pairwise (two-sided) Wilcoxon rank-sum tests at the consumer (market) level for Part II reveal that the differences between High and all the other conditions are significant, but none of the differences between other treatments are statistically significant (see Table F2 in Appendix F).

Table 6: GLS (random-effects) regressions

\begin{tabular}{|c|c|c|c|c|}
\hline & $\begin{array}{c}\text { (1) } \\
\text { Prices } \\
\text { All products }\end{array}$ & $\begin{array}{c}\text { (2) } \\
\text { Markup } \\
\text { All products }\end{array}$ & $\begin{array}{c}\text { (3) } \\
\text { Markup } \\
\text { Harmful product }\end{array}$ & $\begin{array}{c}\text { (4) } \\
\text { Markup } \\
\text { Fair product } \\
\end{array}$ \\
\hline \multirow[t]{2}{*}{ Medium } & 0.244 & 0.819 & 0.450 & 0.876 \\
\hline & (1.073) & $(0.642)$ & $(0.616)$ & $(1.041)$ \\
\hline \multirow[t]{2}{*}{ High } & -0.632 & -1.006 & -0.749 & $-8.004 * * *$ \\
\hline & (1.079) & $(0.702)$ & $(0.776)$ & $(2.106)$ \\
\hline \multirow[t]{2}{*}{ Unequal } & -1.981 & 0.388 & 0.678 & 0.916 \\
\hline & $(1.642)$ & $(0.689)$ & $(0.871)$ & $(1.346)$ \\
\hline \multirow{2}{*}{ Part II } & -0.678 & $-1.218 * *$ & $-1.058 * * *$ & -0.194 \\
\hline & $(0.527)$ & $(0.533)$ & $(0.381)$ & $(0.683)$ \\
\hline \multirow[t]{2}{*}{ Part II x Medium } & 1.725 & 1.328 & 0.284 & 3.366 \\
\hline & $(1.881)$ & (1.559) & $(0.686)$ & $(2.472)$ \\
\hline \multirow[t]{2}{*}{ Part II $\times$ High } & $13.311^{* * *}$ & $9.435^{* * *}$ & $2.946 * * *$ & $17.370 * * *$ \\
\hline & $(2.436)$ & $(2.693)$ & $(0.677)$ & $(3.546)$ \\
\hline \multirow[t]{2}{*}{ Part II x Unequal } & $2.022^{*}$ & 1.404 & 0.021 & 3.151 \\
\hline & $(1.039)$ & $(0.930)$ & $(0.569)$ & (2.348) \\
\hline \multirow[t]{2}{*}{ Constant } & $27.617 * * *$ & $3.604 * * *$ & $3.007 * * *$ & $3.276 * * *$ \\
\hline & $(0.829)$ & $(0.486)$ & $(0.555)$ & $(0.748)$ \\
\hline Observations & 7,469 & 7,469 & 3,080 & 2,362 \\
\hline Number of subjects & 252 & 252 & 211 & 198 \\
\hline
\end{tabular}

Notes. Medium, High and Unequal are binary variables taking on value 1 in the respective treatments and 0 otherwise. Part II is a binary variable taking on value 1 for data from period 11 to 30 and 0 otherwise. Baseline and Part I serve as omitted categories. Models (1) and (2) include all product types. Model (3) focuses on the most harmful ("harmful") product and Model (4) on the least harmful ("fair") product. Standard errors (in parentheses) are clustered at the market level. * significant at $10 \%, * *$ significant at $5 \%, * * *$ significant at $1 \%$.

In order to test the relationships in Figure 4, Model (1) in Table 6 reports random-effects regressions of prices. The coefficients for Part II $x$ Medium, Part II $x$ High, and Part II $x$ Unequal are all positive, but the coefficients on Part II x Medium and Part II x Unequal are small and only the latter is marginally statistically significant. The coefficient for Part II $x$ High is much larger and significant at the one percent level. Thus, a substantial increase in income, from 100 to 400, has a pronounced and significant impact on overall price levels, while a more

\footnotetext{
${ }^{13}$ The average prices across treatments paid in Part I can be found in Table F1 in Appendix F.
} 
moderate increase in (average) income, from 100 to 200, causes directionally identical but small and statistically weak changes.

An increase in prices alone could be due to either consumers buying products that are more socially responsible and costlier to produce or to firms charging higher markups. Therefore, we next consider the markup associated with products sold, i.e., the difference between price and production cost. The average markup paid in Part II across all product types is 2.4 in the Baseline, 4.6 in Medium, 10.8 in High and 4.1 in Unequal. ${ }^{14}$ Products trade with higher markups when consumers have higher incomes but, again, only the differences in markups between High and all other conditions are robustly statistically significant (see Wilcoxon rank-sum tests at the consumer (market) level in Table G2 in Appendix G). Regression Model (2) in Table 6, using markups as the dependent variable, confirms these results. The coefficients for Part II x Medium, Part II x Unequal, and Part II x High are all positive, suggesting that markups increase with income, but the coefficients for Part II $x$ Medium and Part II x Unequal are small and not significant, while the coefficient for Part II $x$ High is much larger and significant at the one percent level. Thus, despite the high levels of firm competition in our design-recall that any of the six firms can sell up to six units, while each of the six consumers can buy at most one unit-firms seem to capture part of the consumers' income growth through higher markups.

Importantly, however, the increased markups are not equivalent across different product types. Figure 5 displays the time path of markups for the two most extreme and most common product types. The most harmful product type, imposing a combined loss of 60, was exchanged in about 41 percent of all cases; the least harmful product type, imposing no loss at all, was exchanged in about 32 percent of all cases. We henceforth refer to these as the "harmful" and "fair" products, respectively. The left panel reveals that the markup for the harmful product differs only slightly across treatments. Markups quickly converge to low levels of about 2.5 in Part I and remain, with some variation in High, at this level throughout Part II. Overall, average markups for the harmful product are very similar across conditions in Part II (1.6 in Baseline, 2.3 in Medium, 3.5 in High, and 2.8 in Unequal). ${ }^{15}$ For the fair product, however, we observe a different pattern of markups in Part II, as shown in the right panel of Figure 5. All three treatments with income increases yield markups for the fair product that are substantially higher than the fair-product markup in the Baseline. The additional markup for the fair product is

\footnotetext{
${ }^{14}$ The average markups across treatments paid in Part I can be found in Table G1 in Appendix G.

${ }^{15}$ The two-sided Wilcoxon rank-sum tests detect only weakly significant treatment differences in Part II, despite quantitatively small differences overall, as shown in Table G4 in Appendix G.
} 
evident in the first few periods of Part II in all three treatments with income increases and becomes even larger over time in High. Compared to Baseline, markups are more than twice as large in Medium and Unequal and more than four times higher in High (3.4 in Baseline, 7.8 in Medium, 14.8 in High, 8.0 in Unequal). ${ }^{16}$

Figure 5: Markup by product type

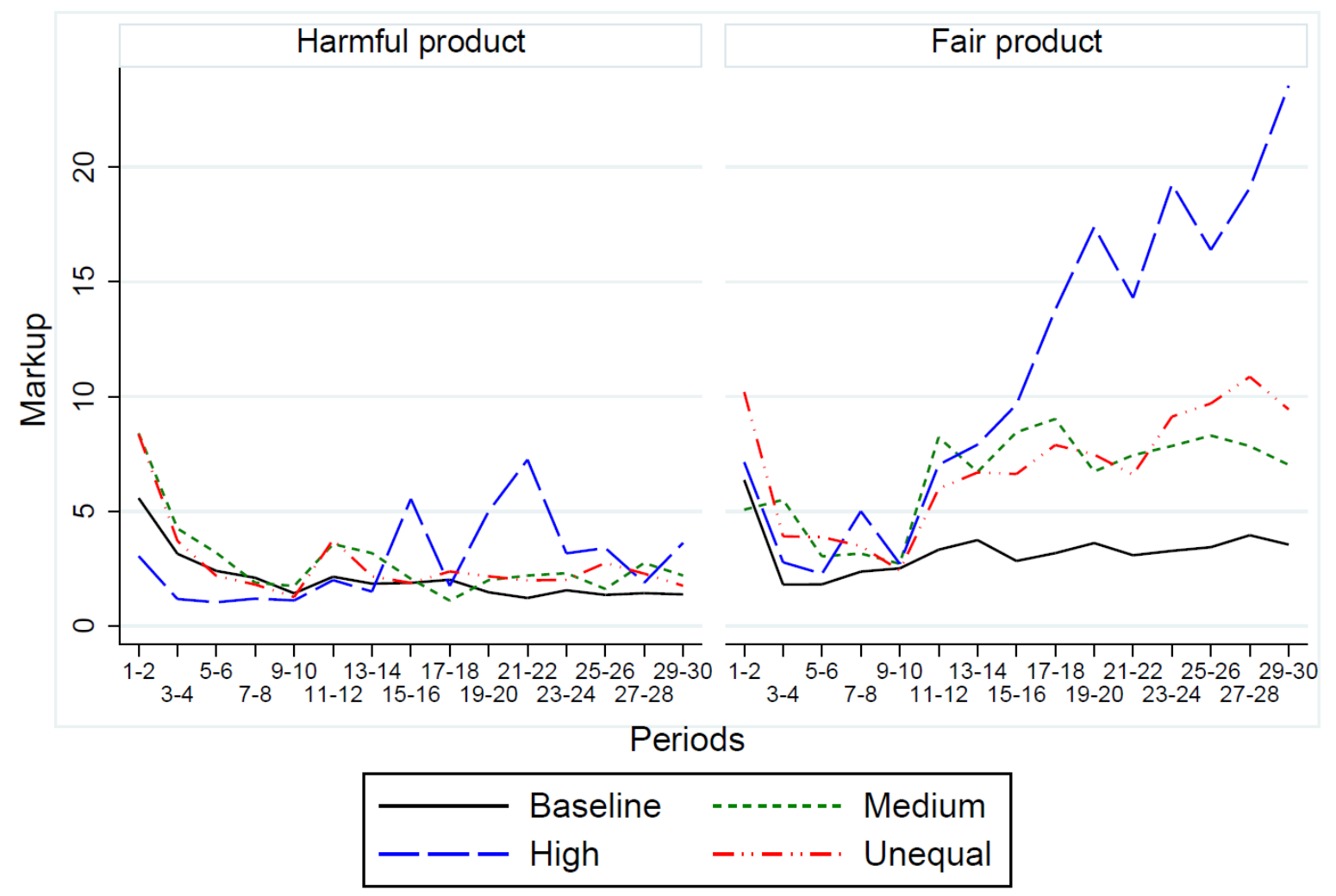

Notes: The "harmful" product imposes a combined loss of 60 on the third parties, and the "fair" product imposes zero loss on the third parties.

The final two models in Table 6 present regressions that study how markups vary by treatment for each product type. Model (3) considers only the harmful product, while Model (4) considers only the fair product. Both models show that the increase in the markup is significant only for High and that the increase is five times as large for the fair product as for the harmful product. That is, there are higher markups when consumers have a substantial increase in income and particularly so for the fair product.

\footnotetext{
16 The differences between Baseline and each of the other treatments are statistically significant at least at the consumer level using two-sided Wilcoxon rank-sum tests (see Table G6 in Appendix G).
} 
While we are cautious not to make too much of these unexpected results regarding prices and markups, the above analysis raises the possibility that firms screen consumers and use the information revealed by responsible consumption choices to establish higher markups for socially responsible products. This observation has implications for how income growth among consumers will translate into market shares of socially responsible products and for the profitability of different kinds of products. It is common knowledge in High that consumers have a substantially higher initial per-period income in Part II than in Part I. Consumers reveal additional information about their social concern by buying socially responsible productsi.e., they reveal not to be entirely self-interested but to have a willingness to pay for avoiding negative impact. Firms apparently exploit this information and capture part of the consumers' income growth by asking for higher markups. Hence, part of the impact of increased social responsibility in response to increased income seems to be captured in higher profit margins for socially responsible products.

\section{Conclusion}

We investigate the relationship between consumers' income and their willingness to voluntarily internalize negative externalities produced by their market behavior, a form of social responsibility. Despite the intuitive appeal that socially responsible market conduct is a normal good, as conjectured by Shleifer (2004) and Bénabou and Tirole (2010), there is a lack of compelling evidence that this is indeed the case. Given the potential social benefits from such voluntary internalization, a positive relationship with income would be important from a policy perspective, as it would dictate that some negative social impacts may be mitigated through voluntary consumer actions as societies experience economic growth.

We begin by documenting that a positive relationship exists in national level shares of expenditures on one kind of socially responsible product—specifically, Fair Trade products— and GDP per capita. The cross-country relationship is strongly positive, suggesting that socially responsible consumption is a normal good. However, there are many other factors that may differ between countries — or even across a single country over time-making causal inference from such data questionable. Nevertheless, this analysis provides suggestive evidence that social responsibility may, in fact, be a normal good.

To obtain causal evidence of the relationship between income and market social responsibility, we conduct a laboratory experiment that creates a stylized product market with varying product types that differ in their degree of negative social impact. More socially responsible products cost more to produce, meaning that consumers have to be willing to pay 
for such products in order for them to be sufficiently profitable for firms to provide. Using this design, we introduce exogenous positive shocks to consumers' incomes in order to identify the resulting effect of such income growth on socially responsible consumption.

Our results corroborate the observation from cross-national comparisons that indicate a positive relationship between income and the willingness to pay for products that mitigate social impact. This effect is always positive in our experiment, though it is only substantial and statistically significant when we implement large increases in consumers' incomes. Nevertheless, the magnitude of these income increases is not implausible, and corresponds to recent ten-year levels of growth in at least some developing countries. Moreover, when we pool all of our treatments and estimate the aggregate effect of income increases, we find a substantial and statistically strong positive effect.

Admittedly, our laboratory evidence comes at the cost of generalizability. Still it is important to document that increased income can yield more socially responsible consumption, which our study achieves in a much more controlled manner than is possible using naturally occurring data. Moreover, similar designs have previously demonstrated concordance between laboratory behavior and purchasing decisions involving real products (Engelmann et al. 2018). We find that such a relationship also exists in our data, using self-reported measures of nonlaboratory purchasing behavior.

Our results also produce additional interesting insights that may be relevant for understanding the consequences of increasing income in real-world product markets involving the possibility of socially responsible production. First, we find little evidence that relative income concerns matter much in determining social responsibility. Consumers respond to income increases fairly consistently, regardless of whether others' incomes also increase. Second, we find an asymmetry in how different kinds of products are marked up in response to consumers' rising income. In particular, firms seem to raise prices considerably more for the most socially responsible types of products and, importantly, consumers are willing to pay these higher prices despite the presence of firm competition. Thus, as consumers become richer, the price for the least socially conscious products does not change much, but the prices for those products with the most positive social impact experience sharp increases, particularly as consumers become much richer. Thus, one consequence of increased consumer income may be greater potential profits for firms selling to the most socially conscious consumers, as these become less price sensitive. However, our experiment provides only limited data in addressing these issues, making it important to explore further the effects of increasing consumers' incomes on socially responsible market outcomes. 


\section{References}

Andreoni, J., 2006. Philanthropy. Handbook of the Economics of Giving, Altruism and Reciprocity, 2, pp.1201-1269.

Andreoni, J. and Bernheim, B.D., 2009. Social image and the 50-50 norm: A theoretical and experimental analysis of audience effects. Econometrica, 77(5), pp.1607-1636.

Andreoni, J., Nikiforakis, N. and Stoop, J., 2017. Are the rich more selfish than the poor, or do they just have more money? A natural field experiment (No. w23229). National Bureau of Economic Research.

Andreoni, J. and Payne, A.A., 2013. Charitable giving. In Handbook of public economics (Vol. 5, pp. 1-50). Elsevier.

Alvaredo, F., Atkinson, A., Piketty, T., and Saez, E., 2013. The Top 1 Percent in International and Historical Perspective. Journal of Economic Perspectives, 27 (3), pp. 3-20.

Bartling, B., Weber, R.A. and Yao, L., 2015. Do markets erode social responsibility? The Quarterly Journal of Economics, 130(1), pp.219-266.

Bartling, B., Valero, V. and Weber, R.A., in press. On the Scope of Externalities in Experimental Markets. Experimental Economics.

Bénabou, R. and Tirole, J., 2010. Individual and corporate social responsibility. Economica, 77(305), pp.1-19.

Bock, O., Baetge, I., and Nicklisch, A., 2014. hroot: Hamburg registration and organization online tool. European Economic Review, 71, pp. 117-120.

Cappelen, A., Halvorsen, T., Sørensen, E., and Tungodden, B., 2017. Face-saving or fairminded: What motivates moral behavior? Journal of the European Economic Association, 15 (3), pp. 540-557.

Carpenter, J., Verhoogen, E. and Burks, S., 2005. The effect of stakes in distribution experiments. Economics Letters, 86(3), pp.393-398.

Cherry, T.L., Frykblom, P. and Shogren, J.F., 2002. Hardnose the dictator. American Economic Review, 92(4), pp.1218-1221.

Danz, D., Engelmann, D. and Kübler, D., 2012. Do legal standards affect ethical concerns of consumers? An experiment on minimum wages (No. 12-3). Working Paper Series, Department of Economics, University of Mannheim.

Engel, C., 2011. Dictator games: A meta study. Experimental Economics, 14(4), pp.583-610.

Engelmann, D., J. Friedrichsen and D. Kübler. 2018. Fairness in markets and market experiments. WZB Discussion Paper SP II 2018-203. 
Fairtrade Labelling Organizations International (FLO), Annual Reports, 2005/06 to 2013/14, accessed January 2017 at https://annualreport16-17.fairtrade.net/en/

Fischbacher, U., 2007. z-Tree: Zurich toolbox for ready-made economic experiments. Experimental economics, 10(2), pp.171-178.

Forsythe, R., Horowitz, J.L., Savin, N.E. and Sefton, M., 1994. Fairness in simple bargaining experiments. Games and Economic behavior, 6(3), pp.347-369.

Friedrichsen, J., 2017. Is Socially Responsible Production a Normal Good? Discussion Papers of DIW Berlin 1644, DIW Berlin, German Institute for Economic Research.

Kirchler, M., Huber, J., Stefan, M. and Sutter, M., 2015. Market design and moral behavior. Management Science, 62(9), pp.2615-2625.

Krupka, E and Weber, R., 2013. Identifying Social Norms Using Coordination Games: Why Does Dictator Game Sharing Vary? Journal of the European Economic Association, 11(3), pp. 495-524.

Piff, P.K., Kraus, M.W., Côté, S., Cheng, B.H. and Keltner, D., 2010. Having less, giving more: the influence of social class on prosocial behavior. Journal of personality and social psychology, 99(5), p.771.

Piff, P.K., Stancato, D.M., Côté, S., Mendoza-Denton, R. and Keltner, D., 2012. Higher social class predicts increased unethical behavior. Proceedings of the National Academy of Sciences, 109(11), pp.4086-4091.

Pigors, M. and Rockenbach, B., 2016. Consumer social responsibility. Management Science, 62(11), pp.3123-3137.

Shleifer, A., 2004. Does competition destroy ethical behavior? American Economic Review, 94(2), pp. 414-418.

Sutter, M., Huber, J., Kirchler, M. and Stefan, M., 2016. Where to Look for the Morals in Markets? IZA Discussion Paper No. 10105.

Vesterlund, L., 2006. Why do people give. The Nonprofit Sector: A Research Handbook, 2, pp. 168-190. 


\section{Appendix}

\section{A. Country Level Data on Income and Consumption of Fair Trade Products}

The 17 graphs below illustrate the correlation between the share of Fair Trade products and the GDP per capita per country from 2004 to 2013. We selected the countries for which we were able to obtain spending data on fair trade products from 2004. As previously, our calculations are based on data from the World Bank database and Fairtrade Labelling Organizations International (FLO), Annual Reports 2005/06 to 2013/14. GDP in 2013 US dollars.

Figure A.1. Austria

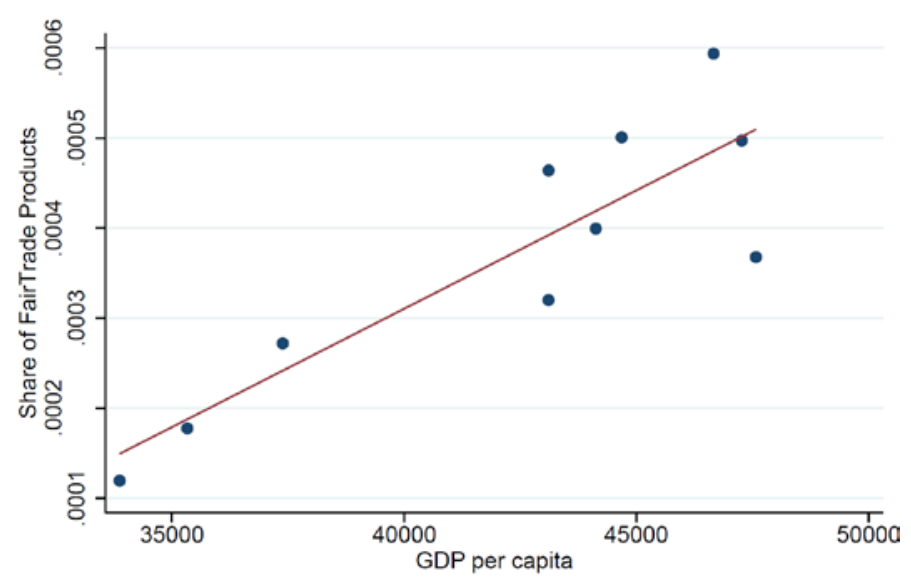

Figure A.3. Canada

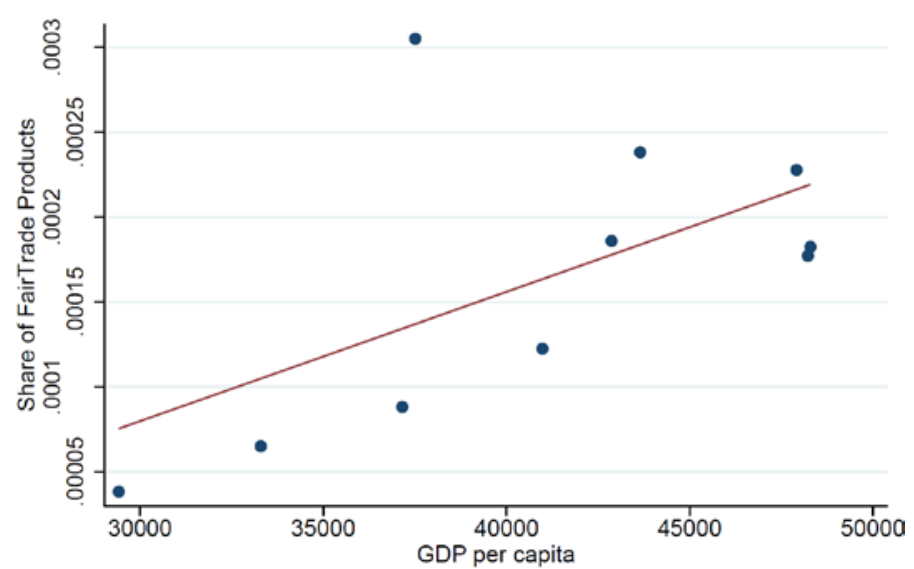

Figure A.2. Belgium

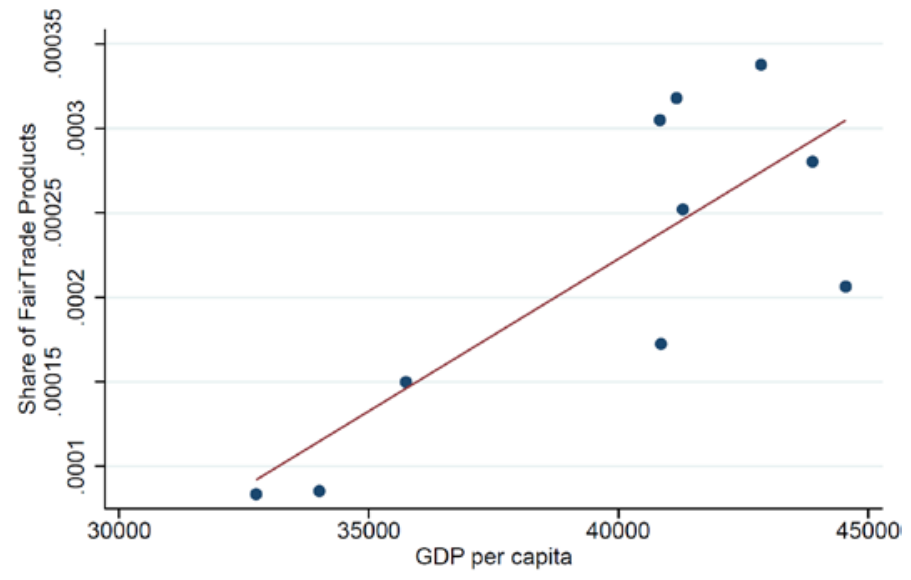

Figure A.4. Denmark

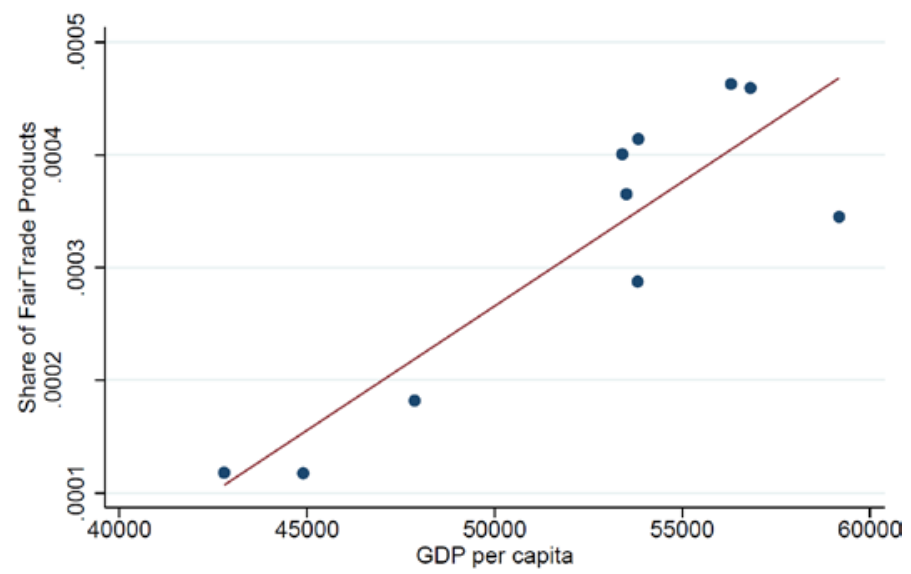


Figure A.5. Finland

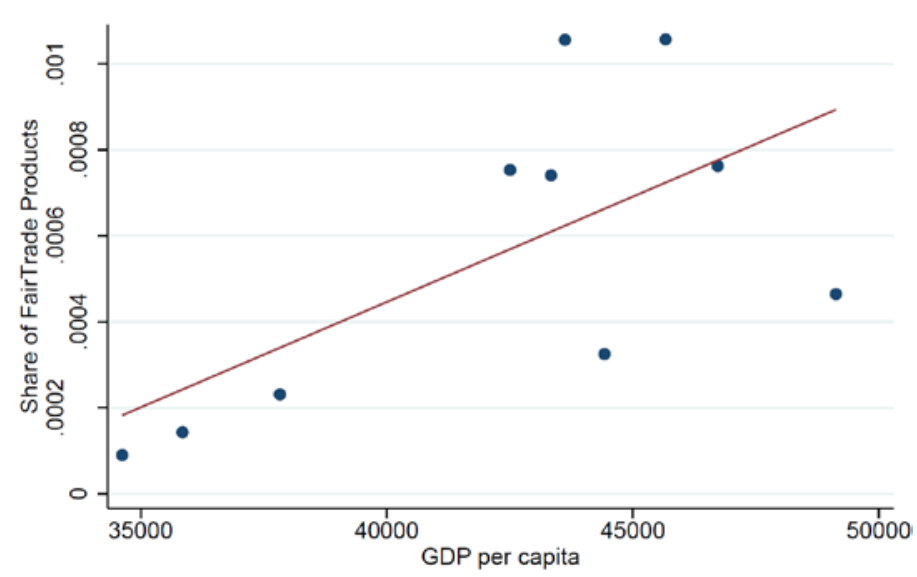

Figure A.7. Germany

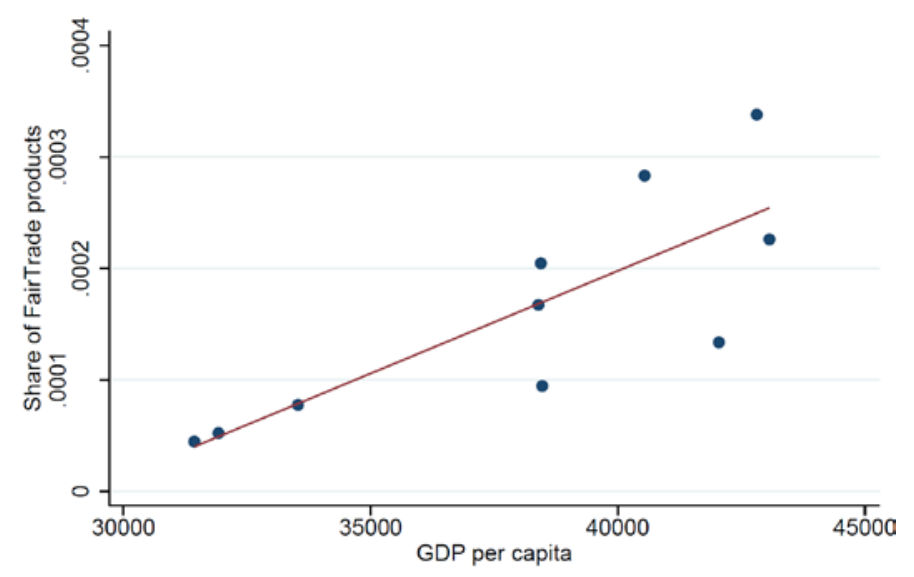

Figure A.9. Ireland

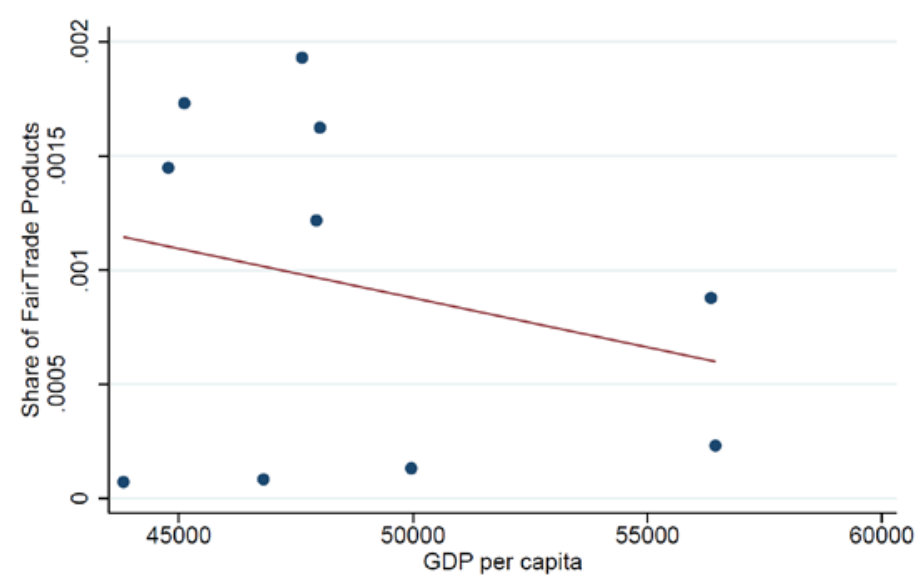

Figure A.6. France

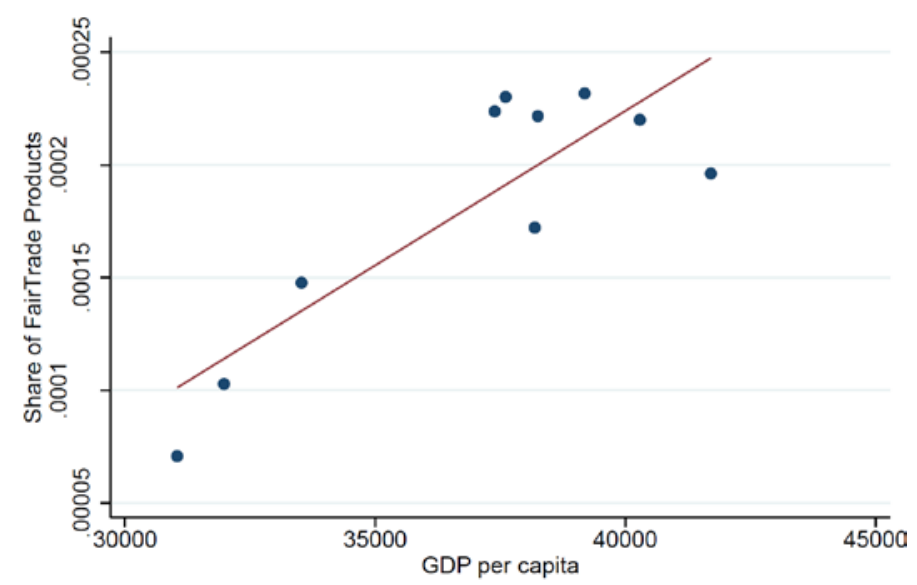

Figure A.8. Italy

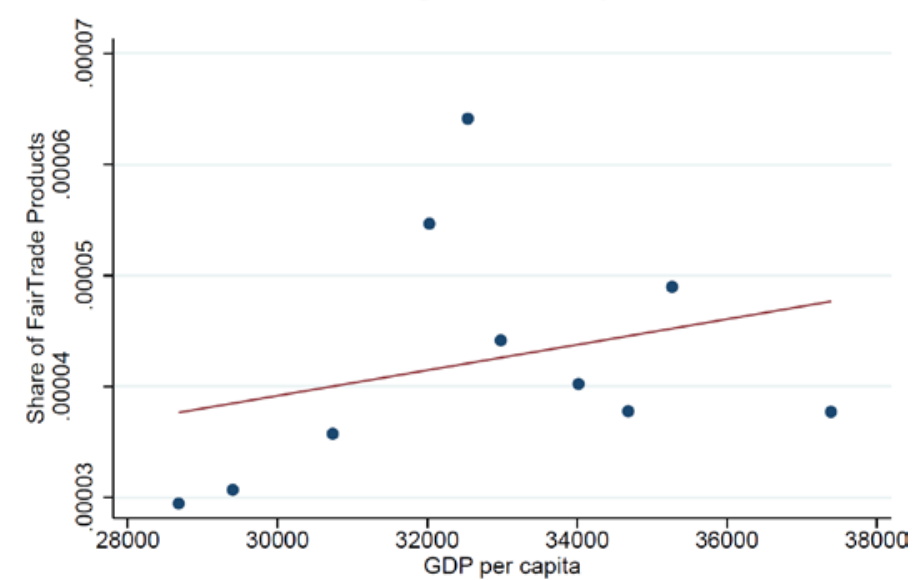

Figure A.10. Japan

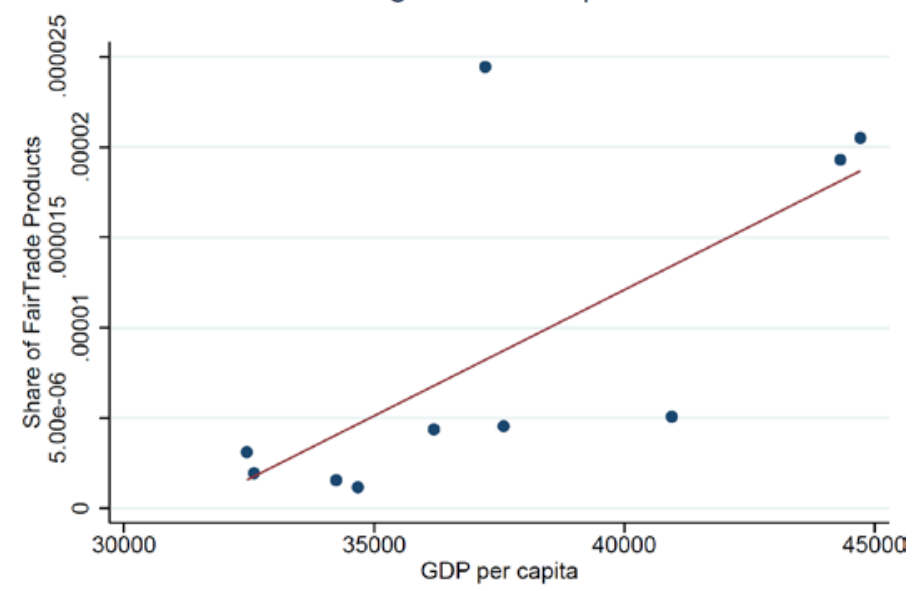


Figure A.11. Luxembourg

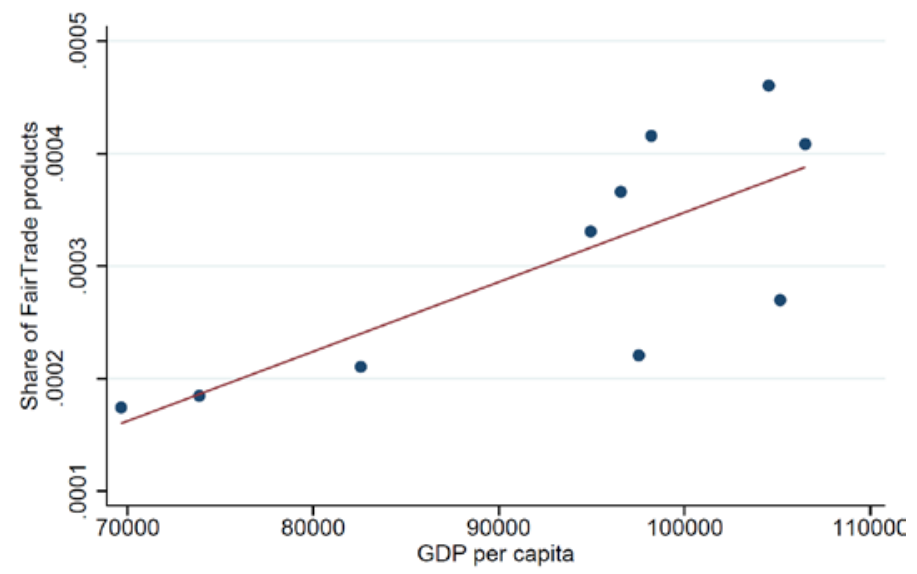

Figure A.13. Norway

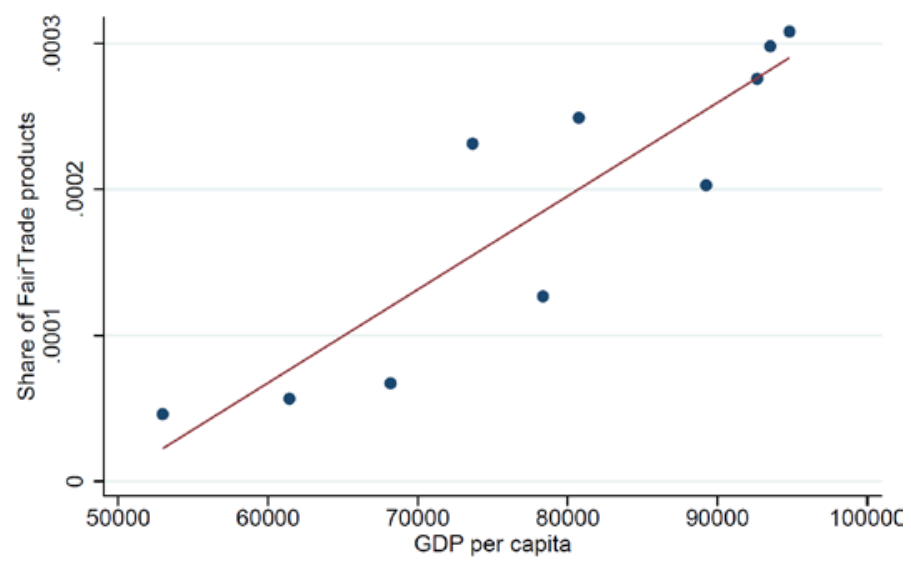

Figure A.15. Switzerland

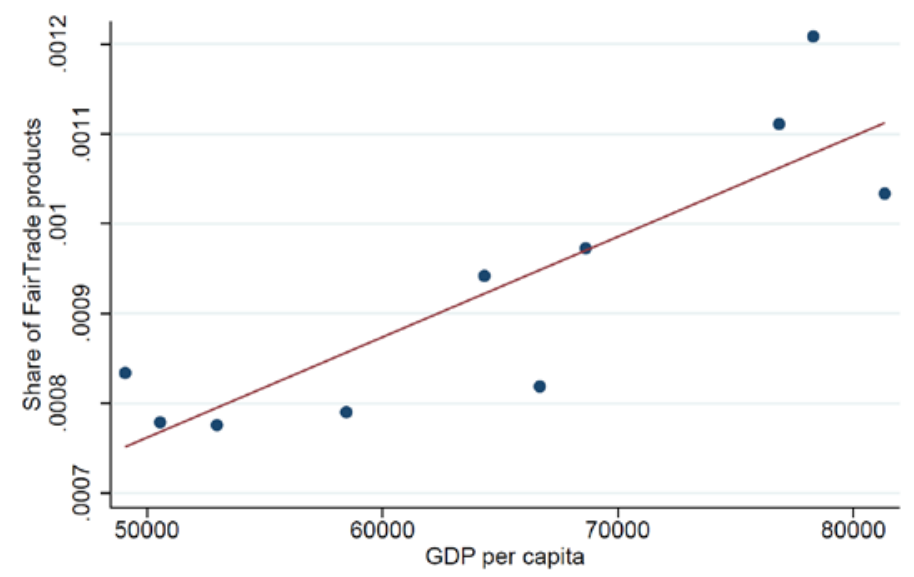

Figure A.12. Netherlands

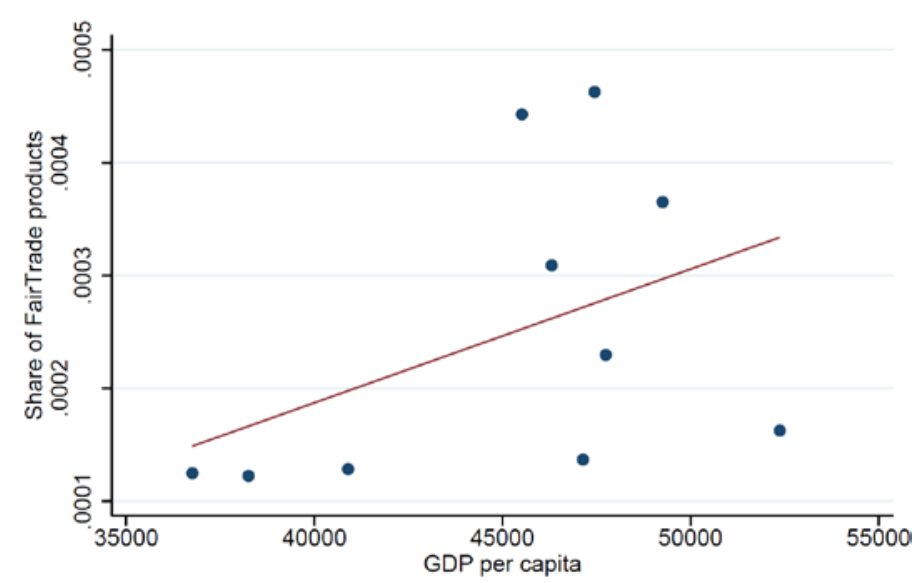

Figure A.14. Sweden

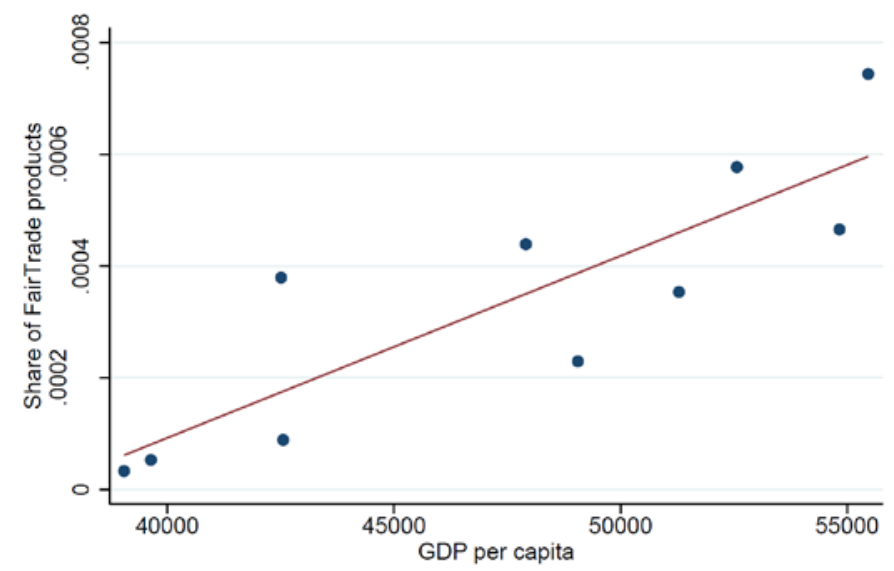

Figure A.16. United Kingdom

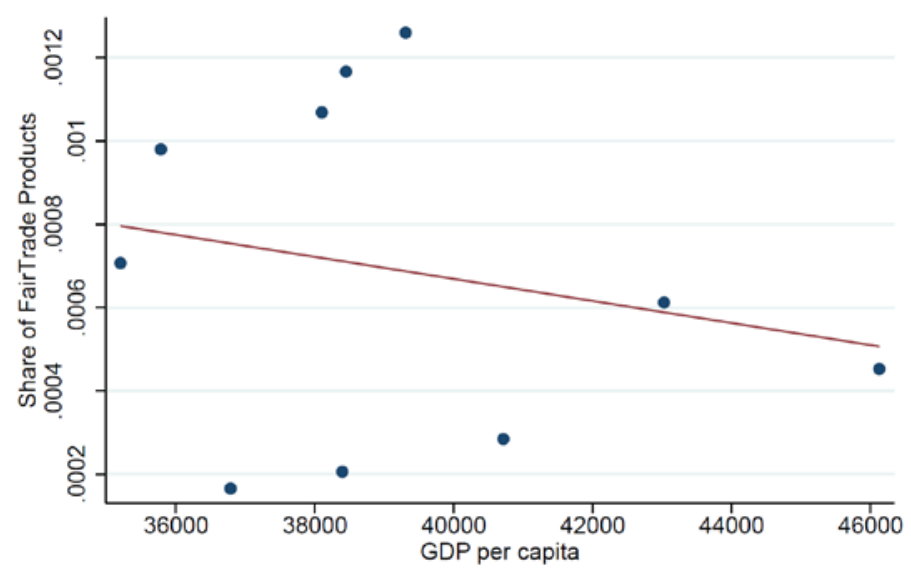


Figure A.17. United States

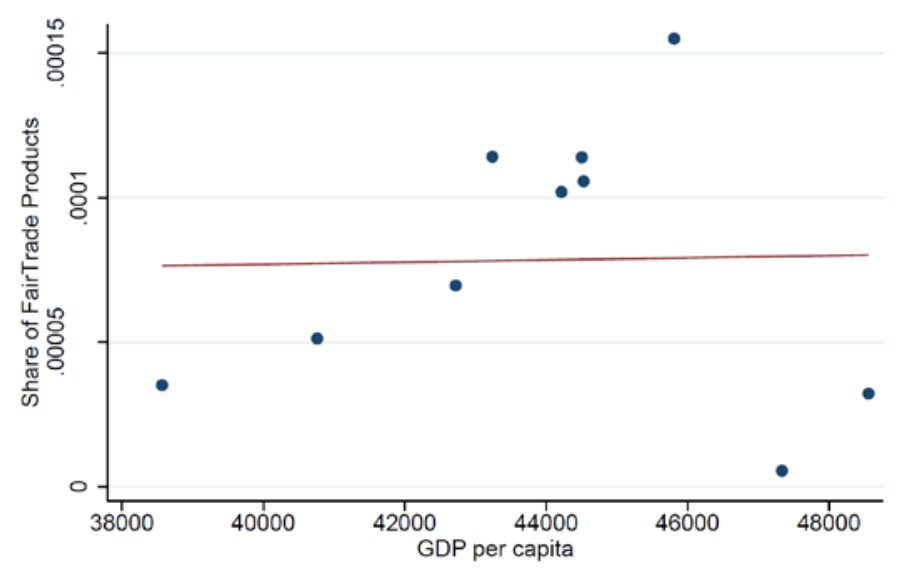




\section{B. Interfaces for Firms' and Consumers' Decisions}

For Firms: They can select the product type they want to sell, and determine a price for their product offer.

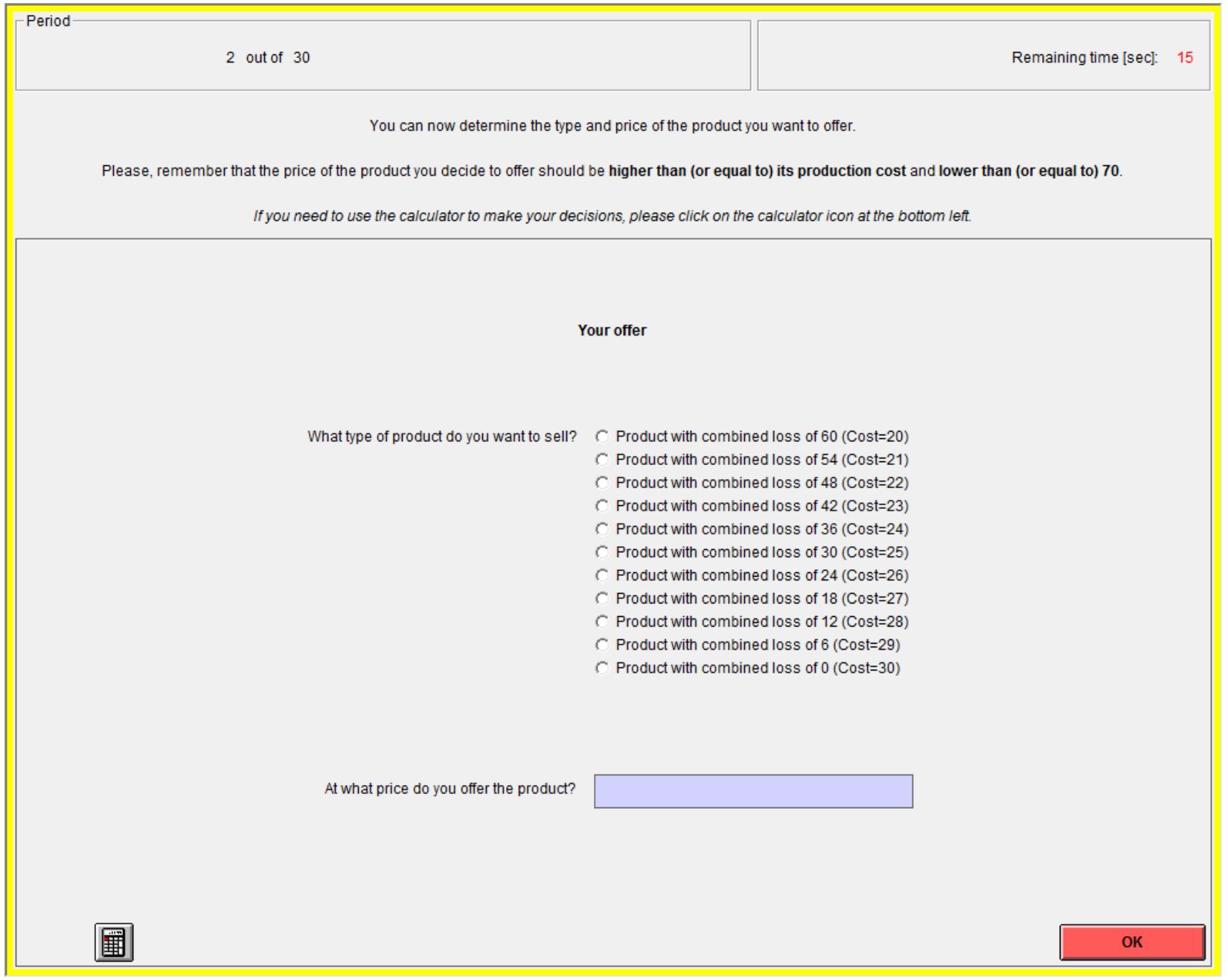


For Consumers: They decide which product they want to buy if any.

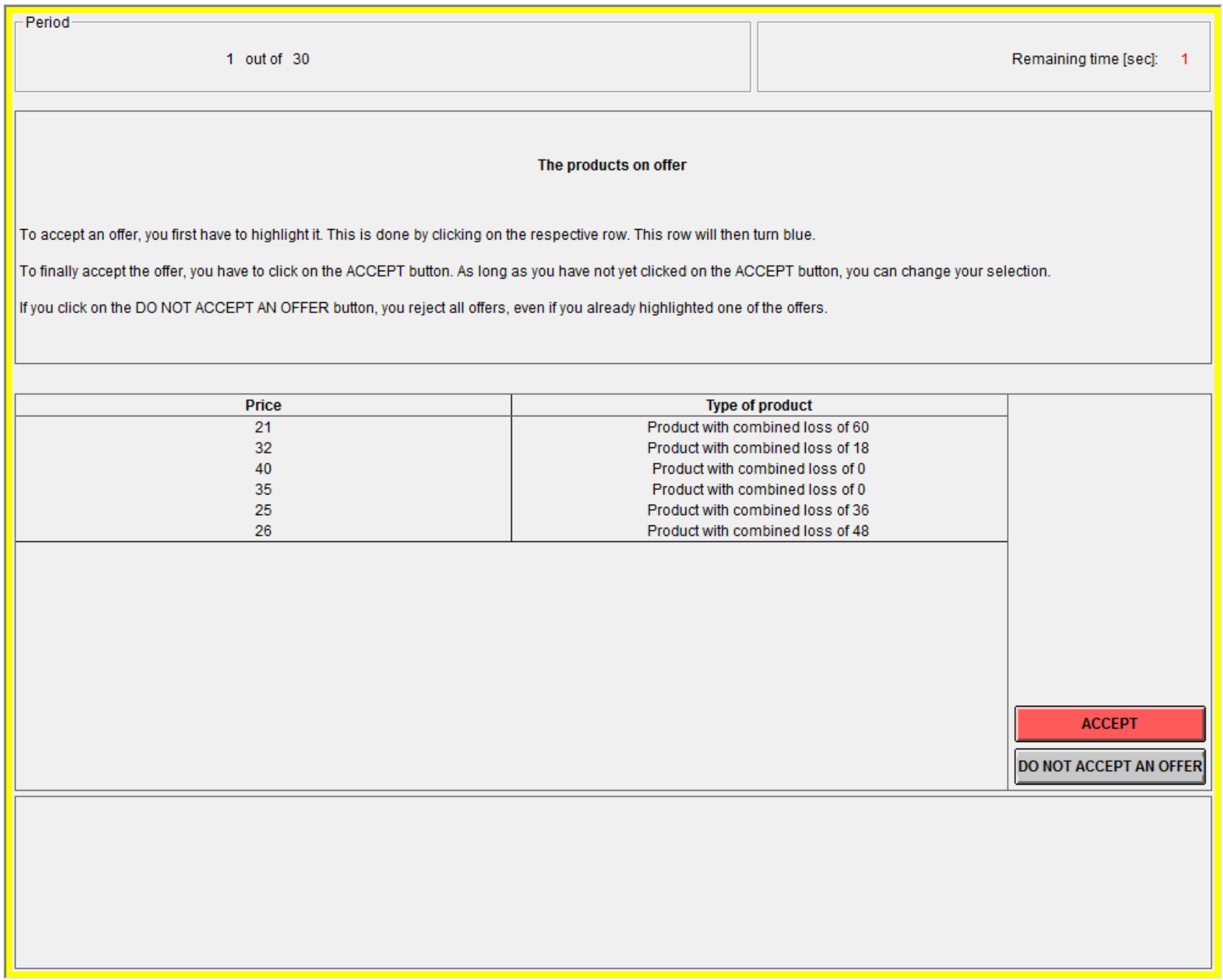




\section{Feedback Received by Participants at the End of Each Period}

For Firms: They observe the offers made by all firms and their respective payments.

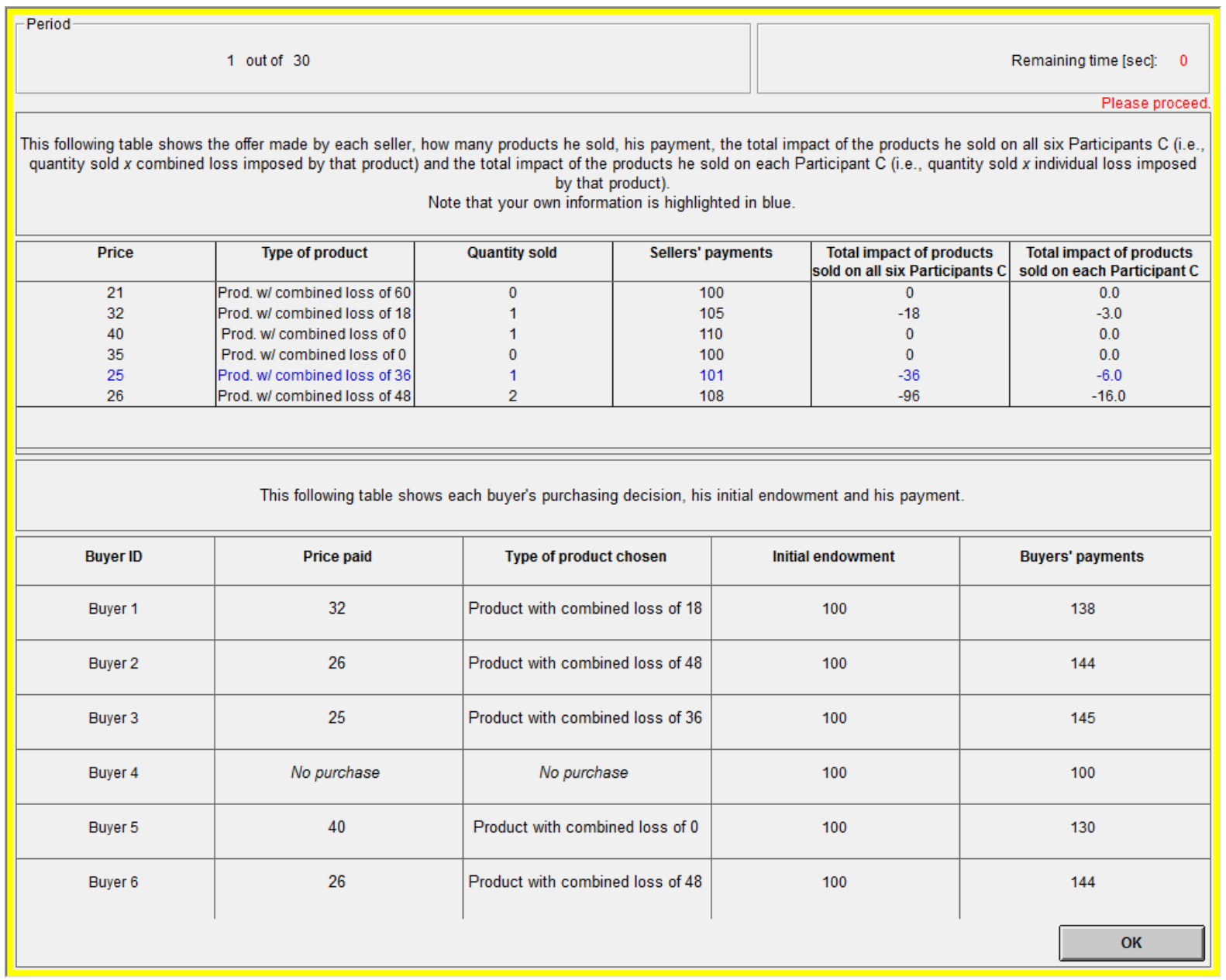


For Consumers: They observe the effect of their purchasing decision on the payments of the third parties.

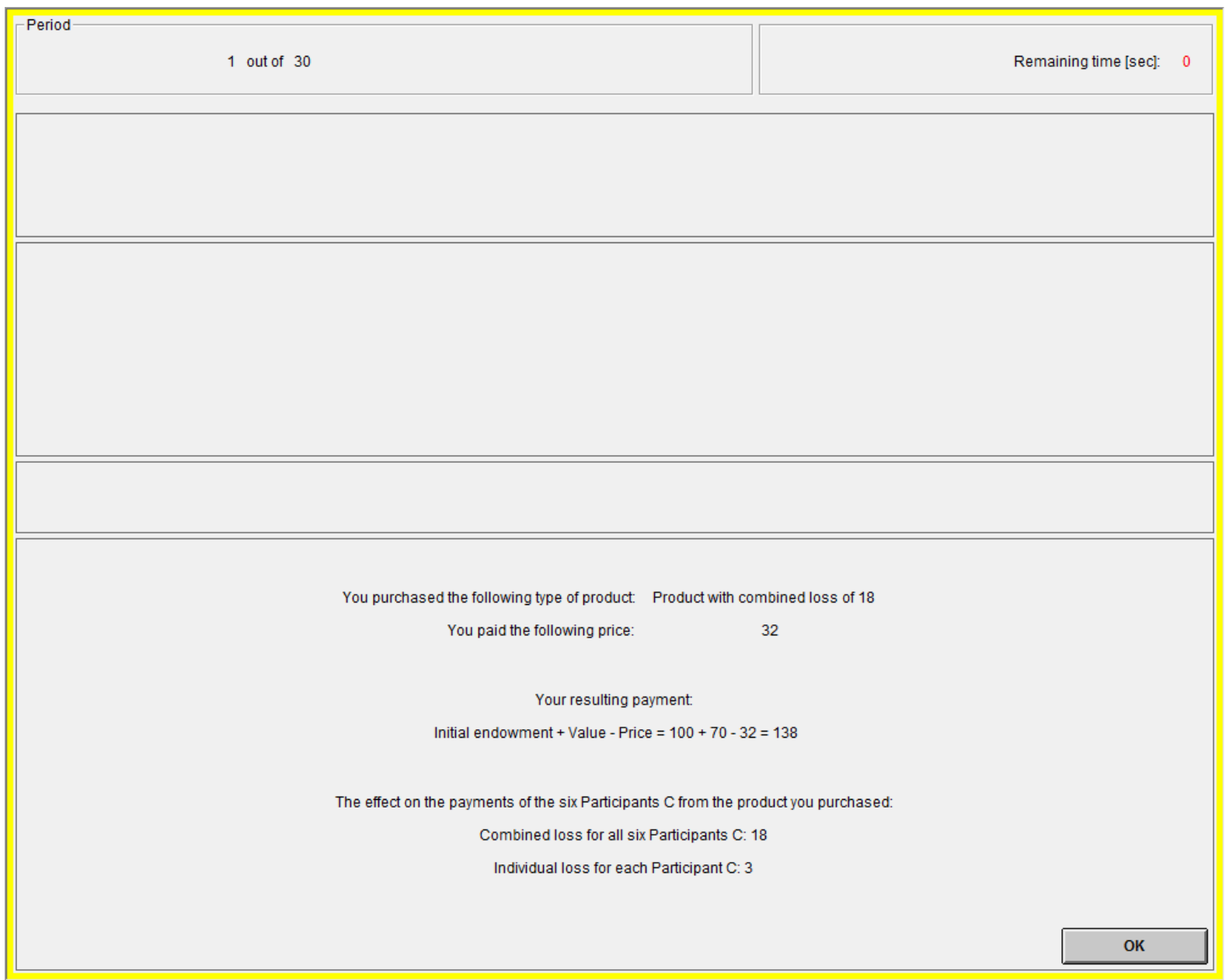


For Third-Parties: They can only observe their payment.

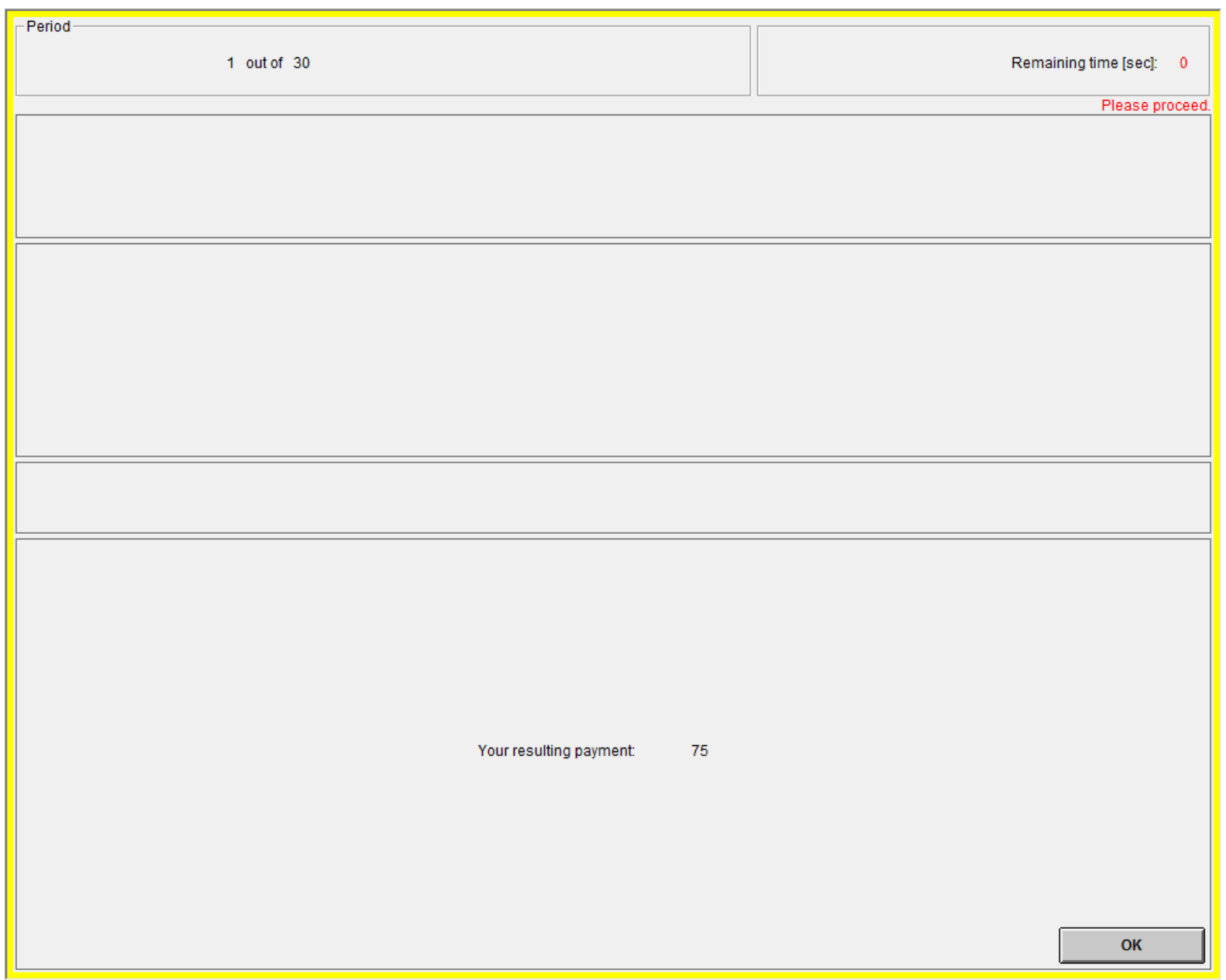




\section{Questionnaire Data}

Table D1: Detailed description of the variables from the questionnaire

\begin{tabular}{|c|c|}
\hline Variables & Description \\
\hline Female & 0- No (Male); 1- Yes \\
\hline Age & In years \\
\hline Study & Field of study 1- Economics, 0- Other \\
\hline $\begin{array}{l}\text { Parents’ } \\
\text { education }\end{array}$ & $\begin{array}{l}\text { What is the highest degree or level of education completed by either of your parents? } \\
0 \text { - don't know / no answer, } 1 \text { - did not complete Medium school, } 2 \text { - Medium school, } \\
3 \text { - some college (i.e. university), } 4 \text { - bachelor's degree, } 5 \text { - master's degree, } 6 \text { - } \\
\text { advanced graduate work or Ph.D. }\end{array}$ \\
\hline $\begin{array}{l}\text { Disposable } \\
\text { income }\end{array}$ & $\begin{array}{l}\text { How much money (CHF) do you have at your disposal each month (approximately, } \\
\text { after housing costs)? }\end{array}$ \\
\hline $\begin{array}{l}\text { Relative } \\
\text { income }\end{array}$ & $\begin{array}{l}\text { How do you think your income and financial situation currently compare to those of } \\
\text { others in Switzerland who are of similar age? } \\
0 \text { - don't know / no answer, } 1 \text { - much below average, } 2 \text { - somewhat below average, } 3 \text { - } \\
\text { about the average, } 4 \text { - somewhat above average, } 5 \text { - much above average }\end{array}$ \\
\hline $\begin{array}{l}\text { Family } \\
\text { income }\end{array}$ & $\begin{array}{l}\text { Approximately, what was the highest total gross income obtained by your parents in } \\
\text { any past year? } \\
0 \text { - don't know / no answer, } 1 \text { - under CHF 50'000, } 2 \text { - CHF 50'000 to 100'000, } 3 \text { - } \\
\text { CHF } 100^{\prime} 000 \text { to CHF } 150^{\prime} 000 \text {, } 4 \text { - CHF } 150^{\prime} 000 \text { to CHF } 200 \text { '000, } 5 \text { - above CHF } \\
200^{\prime} 000\end{array}$ \\
\hline $\begin{array}{l}\text { Responsible } \\
\text { consumption }\end{array}$ & $\begin{array}{l}\text { How often do you buy socially responsible products (e.g. fair trade, sweatshop-free, } \\
\text { environmentally friendly) when such products are available? (select the response that } \\
\text { best describes your behavior): } \\
0 \text { - don't know / no answer, } 1 \text { - never, } 2 \text { - sometimes, } 3 \text { - often, } 4 \text { - always }\end{array}$ \\
\hline $\begin{array}{l}\text { Responsible } \\
\text { purchase }\end{array}$ & $\begin{array}{l}\text { Suppose that you want to buy a jacket. One jacket costs CHF } 100 \text { and it is produced } \\
\text { in a sweatshop (i.e., a factory where manual workers are employed at low wages for } \\
\text { long hours and under poor and often unsafe conditions). How much would you pay } \\
\text { for the exact same jacket but sweatshop-free? }\end{array}$ \\
\hline $\begin{array}{l}\text { Donation to } \\
\text { charity }\end{array}$ & $\begin{array}{l}\text { Approximately, how much money do you donate per year to charitable causes (in } \\
\text { CHF)? }\end{array}$ \\
\hline $\begin{array}{l}\text { Political } \\
\text { orientation }\end{array}$ & $\begin{array}{l}\text { Where would you classify yourself on the left/right political spectrum? } \\
\text { from } 1 \text { (left-wing) to } 9 \text { (right-wing). }\end{array}$ \\
\hline Political party & To which Swiss political party do you feel closest? \\
\hline Work & How many hours do you work per week, alongside your studies (during the semester)? \\
\hline Fair market 1 & The free market system is a fair system.* \\
\hline Fair market 2 & Common or "normal" business practices must be fair, or they would not survive. * \\
\hline Fair market 3 & Acting in response to market forces is not always a fair way to conduct business. ${ }^{*}(-)$ \\
\hline Fair market 4 & In free market systems, people tend to get the outcomes that they deserve. ${ }^{*}$ \\
\hline Fair market 5 & $\begin{array}{l}\text { Profitable businesses tend to be more morally responsible than unprofitable } \\
\text { businesses.* }\end{array}$ \\
\hline Fair market 6 & Economic markets do not fairly reward people.* $\left.{ }^{*}-\right)$ \\
\hline
\end{tabular}


Table D2: Descriptive statistics

\begin{tabular}{|c|c|c|c|c|c|c|}
\hline \multirow{2}{*}{$\begin{array}{l}\text { Variable } \\
\text { Female }\end{array}$} & \multicolumn{2}{|c|}{$\mathrm{N}$} & \multicolumn{2}{|c|}{ Mean } & \multicolumn{2}{|c|}{ SD } \\
\hline & 756 & (252) & 0.53 & $(0.54)$ & 0.50 & $(0.50)$ \\
\hline Age & 756 & (252) & 23.65 & $(23.68)$ & 4.32 & (4.69) \\
\hline Study & 756 & (252) & 0.08 & $(0.08)$ & 0.27 & $(0.27)$ \\
\hline Parents' education & 518 & $(180)$ & 4.64 & $(4.69)$ & 0.95 & $(1.00)$ \\
\hline Disposable income & 733 & (247) & 797.00 & $(829.76)$ & 1257.50 & $(1384.72)$ \\
\hline Relative income & 727 & $(244)$ & 2.15 & $(2.27)$ & 1.02 & $(1.04)$ \\
\hline Family income & 541 & $(181)$ & 2.40 & $(2.54)$ & 1.27 & $(1.23)$ \\
\hline Donation to charity & 752 & $(252)$ & 90.87 & $(93.20)$ & 264.70 & (339.95) \\
\hline Responsible consumption & 718 & $(244)$ & 0.43 & $(0.43)$ & 0.50 & $(0.50)$ \\
\hline Responsible purchase & 753 & $(252)$ & 142.76 & $(146.60)$ & 54.74 & $(60.07)$ \\
\hline Political orientation & 756 & $(252)$ & 4.04 & $(4.09)$ & 1.77 & $(1.74)$ \\
\hline Work & 741 & (250) & 6.93 & $(6.97)$ & 10.62 & (10.69) \\
\hline Fair Market 1 & 756 & (252) & -0.60 & $(-0.36)$ & 2.74 & $(2.69)$ \\
\hline Fair Market 2 & 756 & (252) & -0.70 & $(-0.80)$ & 2.88 & (2.91) \\
\hline Fair Market 3 & 756 & (252) & 2.01 & $(2.14)$ & 2.32 & (2.33) \\
\hline Fair Market 4 & 756 & (252) & -1.41 & $(-1.10)$ & 2.63 & (2.63) \\
\hline Fair Market 5 & 756 & (252) & -1.91 & $(-1.87)$ & 2.54 & (2.67) \\
\hline Fair Market 6 & 756 & (252) & 1.45 & $(1.37)$ & 2.37 & (2.39) \\
\hline Market fairness ${ }^{* *}$ & 756 & (252) & -0.00 & $(0.06)$ & 0.76 & $(0.76)$ \\
\hline
\end{tabular}

${ }^{* *}$ We use a factor analysis on answers to questions about fairness in markets, and create a univariate measure of the extent to which participants believe that markets are fair called Market fairness. Note that Fair Market 3 and Fair Market 6 are reverse coded.

Notes. The descriptive statistics in brackets concern buyers only. For each variable, we excluded participants that did not reply to the question or provided implausible answers. 


\section{E. Social Responsibility}

Table E1: Percentage of the total potential loss mitigated

\begin{tabular}{l|cc}
\hline \hline & Part I & Part II \\
\hline Baseline & $37.95 \%$ & $45.25 \%$ \\
Medium & $34.25 \%$ & $42.86 \%$ \\
High & $29.56 \%$ & $74.54 \%$ \\
Unequal & $29.63 \%$ & $40.64 \%$ \\
\hline
\end{tabular}

Table E2: Wilcoxon rank-sum tests at the consumer (market) level, two-sided

\begin{tabular}{l|cccc}
\hline \hline$p$-values & Baseline & Medium & High & Unequal \\
\hline Baseline & - & $0.8131(0.9397)$ & $0.3117(0.4477)$ & $0.3349(0.5980)$ \\
Medium & $0.8335(0.6501)$ & - & $0.2638(0.3290)$ & $0.3126(0.4292)$ \\
High & $0.0002(0.0393)$ & $0.0000(0.0092)$ & - & $0.6399(0.4610)$ \\
Unequal & $0.4835(0.6732)$ & $0.5804(0.7121)$ & $0.0000(0.0007)$ & - \\
\hline
\end{tabular}

Notes. The p-values in the shaded area correspond to Part I and the rest to Part II. 
Table E3: GLS (random-effects) regression of the percent loss mitigated

\begin{tabular}{|c|c|c|c|c|c|}
\hline & $\overline{(1)}$ & $\overline{(2)}$ & (3) & $(4)$ & $(5)$ \\
\hline Medium & $\begin{array}{l}-0.023 \\
(9.591)\end{array}$ & $\begin{array}{c}3.365 \\
(9.213)\end{array}$ & $\begin{array}{c}-8.772 \\
(10.992)\end{array}$ & $\begin{array}{l}-1.171 \\
(9.744)\end{array}$ & $\begin{array}{c}0.789 \\
(9.386)\end{array}$ \\
\hline High & $\begin{array}{c}-7.667 \\
(12.673)\end{array}$ & $\begin{array}{c}-5.662 \\
(12.731)\end{array}$ & $\begin{array}{c}-9.898 \\
(12.142)\end{array}$ & $\begin{array}{c}-9.633 \\
(12.600)\end{array}$ & $\begin{array}{c}-8.701 \\
(12.888)\end{array}$ \\
\hline Unequal & $\begin{array}{l}-7.712 \\
(9.007)\end{array}$ & $\begin{array}{l}-5.531 \\
(8.905)\end{array}$ & $\begin{array}{c}-8.601 \\
(10.175)\end{array}$ & $\begin{array}{l}-8.259 \\
(9.167)\end{array}$ & $\begin{array}{l}-7.629 \\
(9.023)\end{array}$ \\
\hline Part II & $\begin{array}{l}7.473^{*} \\
(4.124)\end{array}$ & $\begin{array}{l}7.046^{*} \\
(4.046)\end{array}$ & $\begin{array}{c}6.712 \\
(4.862)\end{array}$ & $\begin{array}{l}7.473^{*} \\
(4.124)\end{array}$ & $\begin{array}{l}7.473^{*} \\
(4.124)\end{array}$ \\
\hline Part II x Medium & $\begin{array}{c}2.334 \\
(6.463)\end{array}$ & $\begin{array}{c}2.936 \\
(6.541)\end{array}$ & $\begin{array}{c}5.058 \\
(6.780)\end{array}$ & $\begin{array}{c}2.334 \\
(6.463)\end{array}$ & $\begin{array}{c}2.334 \\
(6.463)\end{array}$ \\
\hline Part II x High & $\begin{array}{c}36.693^{* * * *} \\
(9.629)\end{array}$ & $\begin{array}{c}38.282 * * * \\
(9.558)\end{array}$ & $\begin{array}{c}36.728 * * * \\
(9.522)\end{array}$ & $\begin{array}{c}36.693^{* * *} \\
(9.629)\end{array}$ & $\begin{array}{c}36.693 * * * \\
(9.629)\end{array}$ \\
\hline Part II x Unequal & $\begin{array}{c}3.581 \\
(5.233)\end{array}$ & $\begin{array}{c}4.020 \\
(5.250)\end{array}$ & $\begin{array}{c}4.888 \\
(6.158)\end{array}$ & $\begin{array}{c}3.581 \\
(5.233)\end{array}$ & $\begin{array}{c}3.581 \\
(5.233)\end{array}$ \\
\hline Female & $\begin{array}{c}1.180 \\
(4.416)\end{array}$ & $\begin{array}{c}4.503 \\
(4.229)\end{array}$ & $\begin{array}{l}8.762^{*} \\
(4.862)\end{array}$ & $\begin{array}{l}1.426 \\
(4.515)\end{array}$ & $\begin{array}{c}2.345 \\
(4.264)\end{array}$ \\
\hline Age (log) & $\begin{array}{c}11.224 \\
(13.086)\end{array}$ & $\begin{array}{c}1.299 \\
(13.107)\end{array}$ & $\begin{array}{c}5.194 \\
(15.138)\end{array}$ & $\begin{array}{c}14.875 \\
(14.133)\end{array}$ & $\begin{array}{c}5.879 \\
(13.805)\end{array}$ \\
\hline Disposable income (log) & $\begin{array}{c}-7.203 * * * \\
(2.775)\end{array}$ & & & $\begin{array}{c}-6.628 * * \\
(2.710)\end{array}$ & $\begin{array}{c}-6.344^{* *} \\
(2.915)\end{array}$ \\
\hline Relative income & & $\begin{array}{l}-0.506 \\
(2.196)\end{array}$ & & & \\
\hline Family income & & & $\begin{array}{l}2.988^{*} \\
(1.609)\end{array}$ & & \\
\hline $\begin{array}{l}\text { Responsible } \\
\text { consumption }\end{array}$ & $\begin{array}{c}12.212 * * * \\
(4.121)\end{array}$ & $\begin{array}{c}12.192 * * * \\
(4.093)\end{array}$ & $\begin{array}{c}6.519 \\
(5.265)\end{array}$ & & $\begin{array}{c}12.102^{* * *} \\
(4.280)\end{array}$ \\
\hline Responsible purchase & & & & $\begin{array}{l}0.052^{*} \\
(0.029)\end{array}$ & \\
\hline Donation to charity & $\begin{array}{c}0.017 * * * \\
(0.004)\end{array}$ & $\begin{array}{c}0.015 \\
(0.010)\end{array}$ & $\begin{array}{c}0.012 * * \\
(0.005)\end{array}$ & $\begin{array}{c}0.016^{* * * *} \\
(0.005)\end{array}$ & $\begin{array}{c}0.017 * * * \\
(0.004)\end{array}$ \\
\hline Political orientation & $\begin{array}{c}-2.650 * * \\
(1.288)\end{array}$ & $\begin{array}{l}-2.575^{*} \\
(1.332)\end{array}$ & $\begin{array}{l}-1.293 \\
(1.503)\end{array}$ & $\begin{array}{c}-3.361^{* *} \\
(1.392)\end{array}$ & \\
\hline Market fairness & & & & & $\begin{array}{c}-6.873 * * \\
(2.730)\end{array}$ \\
\hline Constant & $\begin{array}{c}49.807 \\
(42.875)\end{array}$ & $\begin{array}{c}33.135 \\
(45.776)\end{array}$ & $\begin{array}{c}11.976 \\
(51.037)\end{array}$ & $\begin{array}{c}35.874 \\
(44.416)\end{array}$ & $\begin{array}{c}50.060 \\
(44.583)\end{array}$ \\
\hline Observations & 7,170 & 6,930 & 5,160 & 7,170 & 7,170 \\
\hline Number of Subjects & 239 & 231 & 172 & 239 & 239 \\
\hline
\end{tabular}

Notes. Medium, High and Unequal are binary variables taking on the value 1 in the respective treatment, and 0 otherwise. Baseline serves as omitted category in all models. Part II is a binary variable taking on value 1 for data generated from period 11 to 30 and 0 from period 1 to 10 . We excluded participants that either did not reveal their disposable income or reported zero, nor indicated how often they buy fair products. In addition, we excluded participants that did not reply to the question about their relative income in regression 2 or about their family income in regression 3. Standard errors (in parentheses) are clustered at the market level. * significant at $10 \%$, ** significant at $5 \%, * * *$ significant at $1 \%$. 


\section{F. Prices}

Table F1: Average prices paid by consumers

\begin{tabular}{l|cc}
\hline \hline & Part I & Part II \\
\hline Baseline & 27.40 & 26.91 \\
Medium & 27.89 & 28.89 \\
High & 25.46 & 38.27 \\
Unequal & 26.95 & 28.18 \\
\hline
\end{tabular}

Table F2: Wilcoxon rank-sum tests at the consumer (market) level, two-sided

\begin{tabular}{l|cccc}
\hline \hline$p$-values & Baseline & Medium & High & Unequal \\
\hline Baseline & - & $0.5659(0.8206)$ & $0.0179(0.2781)$ & $0.3170(0.6353)$ \\
Medium & $0.3250(0.5967)$ & - & $0.0030(0.1931)$ & $0.0677(0.4606)$ \\
High & $0.0000(0.0034)$ & $0.0000(0.0227)$ & - & $0.0350(0.3379)$ \\
Unequal & $0.6488(0.8330)$ & $0.3909(0.9161)$ & $0.0000(0.0051)$ & - \\
\hline
\end{tabular}

Notes. The p-values in the shaded area correspond to Part I and the rest to Part II.

\section{G. Markups}

a. Markups across all product types

Table G1: Average markups paid by consumers

\begin{tabular}{l|cc}
\hline \hline & Part I & Part II \\
\hline Baseline & 3.42 & 2.36 \\
Medium & 4.44 & 4.56 \\
High & 2.46 & 10.82 \\
Unequal & 3.96 & 4.05 \\
\hline
\end{tabular}


Table G2: Wilcoxon rank-sum tests at the consumer (market) level, two-sided

\begin{tabular}{l|cccc}
\hline \hline p-values & Baseline & Medium & High & Unequal \\
\hline Baseline & - & $0.0013(0.0962)$ & $0.0038(0.1927)$ & $0.1845(0.6352)$ \\
Medium & $0.0089(0.4961)$ & - & $0.0000(0.0227)$ & $0.0150(0.3703)$ \\
High & $0.0000(0.0067)$ & $0.0001(0.0227)$ & - & $0.0002(0.0708)$ \\
Unequal & $0.1628(0.6732)$ & $0.3509(0.9790)$ & $0.0000(0.0223)$ & - \\
\hline
\end{tabular}

Notes. The p-values in the shaded area correspond to Part I and the rest to Part II.

\section{b. Markups for "harmful” products}

Table G3: Average markups paid for the "harmful” products by consumers

\begin{tabular}{l|cc}
\hline & Part I & Part II \\
\hline Baseline & 2.79 & 1.64 \\
Medium & 3.63 & 2.28 \\
High & 1.61 & 3.52 \\
Unequal & 3.31 & 2.83 \\
\hline
\end{tabular}

Table G4: Wilcoxon rank-sum tests at the consumer (market) level, two-sided

\begin{tabular}{l|cccc}
\hline \hline$p$-values & Baseline & Medium & High & Unequal \\
\hline Baseline & - & $0.0291(0.2899)$ & $0.0012(0.1931)$ & $0.5890(0.8330)$ \\
Medium & $0.0714(0.4963)$ & - & $0.0000(0.0301)$ & $0.1116(0.4291)$ \\
High & $0.0006(0.0979)$ & $0.0366(0.4624)$ & - & $0.0003(0.1215)$ \\
Unequal & $0.8643(1.0000)$ & $0.1668(0.4605)$ & $0.0070(0.2149)$ & - \\
\hline
\end{tabular}

Notes. The p-values in the shaded area correspond to Part I and the rest to Part II. 


\section{c. Markups for “fair” products}

Table G5: Average markups paid for the "fair" products by consumers

\begin{tabular}{l|cc}
\hline \hline & Part I & Part II \\
\hline Baseline & 2.83 & 3.40 \\
Medium & 3.63 & 7.80 \\
High & 3.64 & 14.83 \\
Unequal & 4.04 & 8.00 \\
\hline
\end{tabular}

Table G6: Wilcoxon rank-sum tests at the consumer (market) level, two-sided

\begin{tabular}{l|cccc}
\hline \hline$p$-values & Baseline & Medium & High & Unequal \\
\hline Baseline & - & $0.7051(1.0000)$ & $0.2625(0.9024)$ & $0.5052(0.9012)$ \\
Medium & $0.0029(0.4497)$ & - & $0.4747(0.7133)$ & $0.7375(0.9259)$ \\
High & $0.0000(0.0067)$ & $0.0265(0.1037)$ & - & $0.6764(0.7673)$ \\
Unequal & $0.0129(0.2463)$ & $0.4463(0.9161)$ & $0.0027(0.0553)$ & - \\
\hline
\end{tabular}

Notes. The p-values in the shaded area correspond to Part I and the rest to Part II. 


\section{H. Experimental Instructions}

In the following, we provide the instructions for Part I, and the instructions for Part II for the treatments Baseline, Medium, High and Unequal. 


\section{General instructions}

We are pleased to welcome you to this economic study.

Please read the following instructions carefully. You can-depending on your decisions and/or those of the other participants-earn money in addition to the $\mathbf{1 5}$ Swiss francs that you receive as an initial participation payment. It is thus very important that you read the instructions carefully.

This study will have two parts. Part I lasts 10 periods and Part II lasts 20 periods. The entire study will thus last for $\mathbf{3 0}$ periods.

In addition to the initial participation payment of 15 Swiss francs, you will be paid your earnings from one randomly selected period. That is, the computer will randomly select one period out of 30 at the end of the study to count for payment. Since you do not know which period the computer will randomly select, you must consider your decisions in each of the 30 periods very carefully.

During the study, we will not speak of Swiss francs, but of points. The points you earn during the study will be converted to Swiss francs at the end of the study. The following conversion rate applies:

$$
6 \text { points }=2 \text { Swiss francs }
$$

At the end of today's study, you will receive your payment in cash.

We will explain the exact procedures for Part I on the next pages. You will receive instructions about all changes, if any, to the procedures prior to the start of Part II. Note that the decisions taken in Part I of the study do not influence the procedures for Part II.

Communication with the other participants is strictly forbidden during the study. Violation of this rule will lead to exclusion from the study and loss of all payments.

For the sake of simplicity, we will always use male forms of pronouns for participants; the instructions obviously also refer to female participants. 


\section{A. THE MARKET ACTIVITY}

In all periods in Parts I and II, you will participate in the same market activity, described below.

\section{Market participants}

There are three types of participants in this study: Participants A, B, and C. Participants A are sellers and Participants $B$ are buyers. Participants $C$ can neither sell nor buy, but they can incur losses due to the transactions between Participants A and $B$.

The participants are divided into groups of 18 people. There are six Participants A (sellers), six Participants B (buyers), and six Participants C in each group.

You will see whether you are Participant A, B, or C on your screen at the beginning of the study. Your role as Participant A, B, or $\mathrm{C}$ will remain the same during the entire study.

\section{Market products}

In the market activity, different types of product can be exchanged, i.e. sold and bought. The type of product refers to the loss that exchanging a product imposes on Participants C. A product only creates a loss when it is sold by a Participant A (seller) to a Participant B (buyer).

Each possible type of product produces a particular loss for the six Participants $\mathrm{C}$, as shown in Table 1. The individual loss is the loss that the product imposes on each Participant C. This can be any value from 0 to 10 . Because there are six Participants $C$ who all incur the same loss from a product, the total combined loss produced by a product corresponds to the individual loss multiplied by six, and is between 0 and 60 . For example, if the individual loss from a product is 5 points for each of the six Participants $C$ then the combined loss produced by this product is $6 x 5=30$ points.

Each type of product entails a production cost for Participant A when the product is sold. The production cost is between 20 and 30 points and depends on the type of product. Lower production costs imply higher losses for Participants C. Specifically, each decrease in the production cost of 1 point induces a 1-point increase in the individual loss for each Participant C (and, hence, a 6-point increase in the combined loss for all Participants C). Table 1 shows the exact production cost for each type of product.

All products are worth 70 points to Participants B (buyers) when they are bought, regardless of what type of product it is.

Please take a moment to look over the following table carefully. It is important for understanding how earnings in this study are determined. 
Table 1: Types of products and corresponding production costs

\begin{tabular}{cccc}
\hline \hline \multicolumn{2}{c}{$\begin{array}{c}\text { Type of product } \\
\text { (Combined and individual losses for Cs produced by this product) }\end{array}$} & $\begin{array}{c}\text { Production } \\
\text { cost }\end{array}$ \\
\hline \hline Combined loss of $\mathbf{6 0}$ & $\rightarrow$ & Individual loss of $\mathbf{1 0}$ & $\mathbf{2 0}$ \\
\hline Combined loss of $\mathbf{5 4}$ & $\rightarrow$ & Individual loss of $\mathbf{9}$ & $\mathbf{2 1}$ \\
\hline Combined loss of $\mathbf{4 8}$ & $\rightarrow$ & Individual loss of $\mathbf{8}$ & $\mathbf{2 2}$ \\
\hline Combined loss of $\mathbf{4 2}$ & $\rightarrow$ & Individual loss of $\mathbf{7}$ & $\mathbf{2 3}$ \\
\hline Combined loss of $\mathbf{3 6}$ & $\rightarrow$ & Individual loss of $\mathbf{6}$ & $\mathbf{2 4}$ \\
\hline Combined loss of $\mathbf{3 0}$ & $\rightarrow$ & Individual loss of $\mathbf{5}$ & $\mathbf{2 6}$ \\
\hline Combined loss of $\mathbf{2 4}$ & $\rightarrow$ & Individual loss of $\mathbf{4}$ & $\mathbf{2 7}$ \\
\hline Combined loss of $\mathbf{1 8}$ & $\rightarrow$ & Individual loss of $\mathbf{3}$ & $\mathbf{2 8}$ \\
\hline Combined loss of $\mathbf{1 2}$ & $\rightarrow$ & Individual loss of $\mathbf{2}$ & $\mathbf{2 9}$ \\
\hline Combined loss of $\mathbf{6}$ & $\rightarrow$ & Individual loss of $\mathbf{1}$ & $\mathbf{3 0}$ \\
\hline Combined loss of $\mathbf{0}$ & $\rightarrow$ & Individual loss of $\mathbf{0}$ & \\
\hline \hline
\end{tabular}

\section{Market procedures}

- Each Participant A (seller) can make one sales offer in each period, by entering it on the following screen:

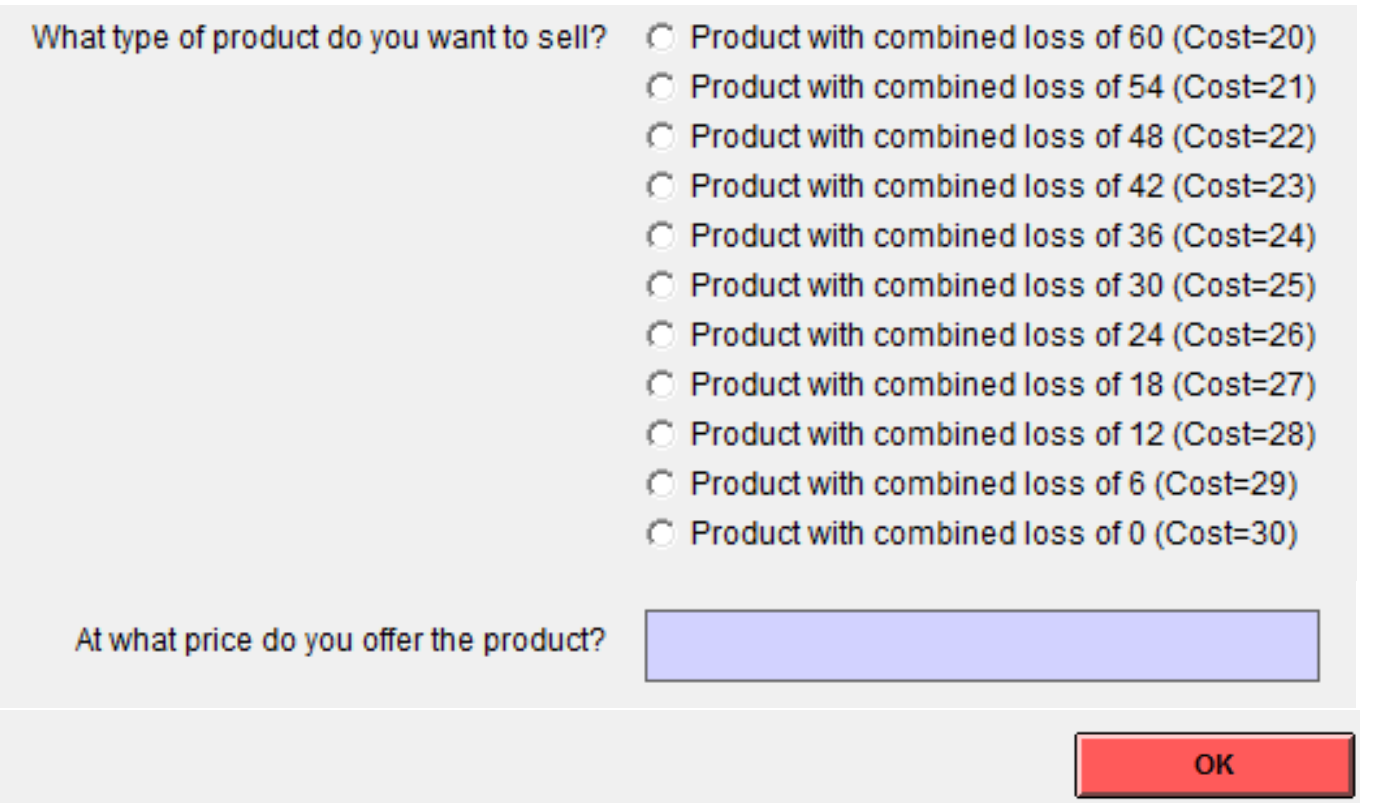


More specifically, each Participant A must indicate:

- The type of product he would like to offer. To do this, he must click on the corresponding type of product.

- $\quad$ The price of the product. The corresponding number must be entered in the box. The price may be any integer between the production cost of the chosen product up to a maximum of 70 .

Once a Participant A has made his decisions, he must click on the OK button at the bottom right of the screen. Note that the type of product and the price can be changed until the OK button is clicked.

Once all six Participants A have made their sales offers, they will be informed about the sales offers (price and type of product) made by all Participants A. This information will be on a screen like the one below:

\begin{tabular}{|c||c||c|}
\hline Price & Type of product & Quantity of products sold \\
\hline $\begin{array}{c}\text { This is where Participants A see } \\
\text { the price of the product for } \\
\text { every sales offer }\end{array}$ & {$\left[\begin{array}{c}\text { This is where Participants A see the } \\
\text { type of product for every sales offer }\end{array}\right.$} & $\begin{array}{c}\text { This is where Participants A } \\
\text { see the quantity of products } \\
\text { sold for every sales offer }\end{array}$ \\
\hline
\end{tabular}

A participant's own sales offer is always marked in blue. In the column on the right, Participants A will see how many Participants B (buyers) accept each of the offers, i.e. the quantity of the product sold by each of the six Participants A.

Each Participant B will make his decision by selecting from one of the six offers, or deciding not to purchase a product. A maximum of 6 products can thus be sold in a given period. These products can be sold by the same or by different Participants A. Therefore, each Participant A can sell between 0 and 6 products in a period.

Once all Participants B have made their decisions, each Participant A will learn the payments of all Participants A. Participants A will also be informed about each Participant B's purchasing decisions and payments. Finally, Participants A will learn the losses incurred by Participants C due to the product(s) he sold. 
- Each Participant B (buyer) can decide whether or not to accept at most one offer. In each period, they can see the six sales offers on a screen like the one below:

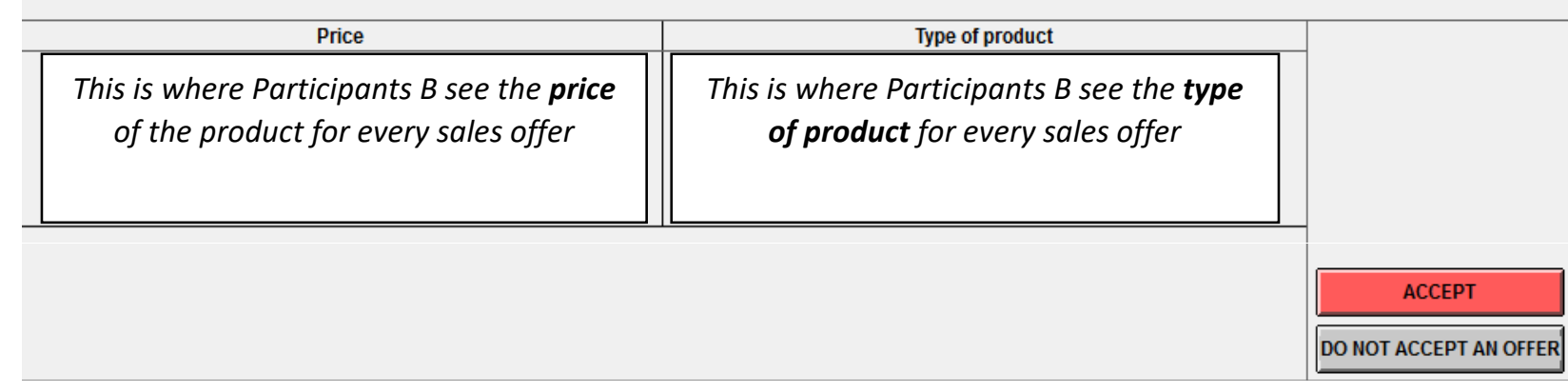

The prices appear in the left column of the table, and the type of product appears in the right column. Each offer is always in a separate row.

- If a Participant B wants to accept an offer, he must first click on the corresponding row. The marked row will then appear with a blue background. In order to accept the offer marked in blue, Participant B must click on the ACCEPT button. Note that the choice of offer can be changed until the ACCEPT button is clicked.

- If a Participant B does not want to accept any offer, he must click on the DO NOT ACCEPT AN OFFER button. Note that even if a row had already been marked, all offers will be declined if the DO NOT ACCEPT AN OFFER button is clicked.

When all Participants B have made their decisions, each Participant B will learn of his own payment and the corresponding losses incurred by Participants $\mathrm{C}$ based on his decision.

- Participants C cannot make any decisions during this study. We ask Participants C, however, to indicate in each period their expectations about the behaviors of Participants A (sellers) and B (buyers).

When all Participants A and B have made their decisions, Participants C will learn of their own payments, which are entirely dependent on the decisions of Participants A and B. These payments depend on the type of products exchanged: Each time a product is exchanged, each of the six Participants $C$ incurs the associated individual loss from that product, which is between 0 and 10 points. Since up to 6 products can be exchanged, the sum of the individual losses incurred by each Participant $C$ is between 0 and 60 points.

After all participants have been informed about their payments in a period, the next period will begin.

Note that, during each period, you will see a timer in the top right corner of your screen. Please use the time indicated by the timer to make your decision. 


\section{B. PAYMENTS OF PARTICIPANTS}

In each period, each Participant A, B, and C initially receives an endowment of 100 points. The payments in points of Participant A (seller), Participant B (buyer), and Participant C in a period are then determined as follows:

Participant A’s payment:

$$
100+\text { quantity sold } x \text { (price - production cost) }
$$

where the production cost, between 20 and 30, depends on the type of product offered, as shown in Table 1.

Note: if no Participant B accepts Participant A’s sales offer, A’s payment is $\mathbf{1 0 0 .}$

Participant B’s payment:

- If Participant B makes a purchase:

$$
100+70-\text { price }
$$

- $\quad$ If Participant B does not make a purchase, his payment is $\mathbf{1 0 0 .}$

- Participant C’s payment:

\section{0 - sum of individual losses}

where the sum of individual losses is the sum of the individual losses for a Participant $\mathrm{C}$ resulting from all products that are exchanged.

Note: if all six Participants B purchase a product with the smallest possible individual loss (0) for Participants C or do not purchase a product, Participants C's payment is 100; if all six Participants B purchase a product with the largest possible individual loss (10) for each Participant C, Participant C’s payment is $\mathbf{4 0 .}$ 


\section{EXAMPLES}

\section{Example 1}

Assume that a Participant A (seller) offers a product with a combined loss of 30 points (i.e. an individual loss of 5 points) at the price of 40 and all six Participants B (buyers) accept this offer. The following payments will result:

- A product with a combined loss of 30 points for Participants $C$ costs 25 points to produce (see Table 1 on Page 3). The Participant A's payment is thus equal to:

$100+$ quantity sold $\boldsymbol{x}($ price - production cost $)=100+6 \boldsymbol{x}(40-25)=190$

- Each Participant B purchased a product. Therefore, each Participant B's payment is equal to:

$100+70-$ price $=100+70-40=130$

- When a product with a combined loss of 30 points is sold, it imposes an individual loss of 5 points on each of the six Participants C. Since six products are sold, the sum of the individual losses for a Participant $C$ is equal to 30 points $(6 x$ 5). Each Participant C's payment is thus equal to:

$100-$ sum of individual losses $=100-6 \times 5=100-30=70$.

\section{- Example 2}

Assume that four Participants B (buyers) accept an offer for a product with a combined loss of 18 points (i.e. individual loss of 3 points). The remaining two Participants B accept an offer for a product with a combined loss of 42 points (i.e. individual loss of 7 points). Here we focus on the payments to each Participant C:

When a product with a combined loss of 18 points is sold, it imposes an individual loss of 3 points on each of the six Participants C. Since four products of this type are sold, each Participant $C$ thus incurs a loss of 12 points $(4 \times 3)$ from these products.

In addition, when a product with a combined loss of 42 points is sold, it imposes an individual loss of 7 points on each of the six Participants C. Since two products of this type are sold, each Participant $C$ thus incurs a loss of 14 points $(2 \times 7)$ from these two products.

Each Participant C's payment is thus equal to:

$100-$ sum of individual losses $=100-\left(4 x_{3}\right)-(2 \times 7)=100-12-14=74$ 


\section{Instructions for Part II}

\section{A. THE MARKET GAME}

As previously announced, the market activity remains the same in Part II.

- Participants A are sellers. At the beginning of each period, each Participant A makes an offer to sell a product. To do so, he has to determine the price and the type (i.e. the losses for Participants C) of the product he would like to offer.

Participants B are buyers. Each Participant B can then choose to buy one product from one of the Participants A, or can choose not to buy a product.

Participants C can neither sell nor buy, but they can incur losses due to the transactions between Participants A and B.

Your role in Part II is the same as it was in Part I.

\section{B. PAYMENTS OF PARTICIPANTS}

In Part II, the way payments are determined by the market activity is the same as in Part I.

In each period, each Participant A, B, and C initially receives an endowment of 100 points as in Part I.

The payments of Participant A (seller), Participant B (buyer), and Participant C in a period are thus determined as follows: 
- Participant A's payment:

$$
100+\text { quantity sold } x \text { (price - production cost) }
$$

where the production cost, between 20 and 30, depends on the type of product offered, as shown in Table 1.

Note: if no Participant B accepts Participant A’s sales offer, Participant A’s payment is 100.

Participant B's payment:

- If Participant B makes a purchase:

$$
100+70-\text { price }
$$

- If Participant B does not make a purchase, his payment is $\mathbf{1 0 0 .}$

- Participant C’s payment:

$$
100 \text { - sum of individual losses }
$$

where the sum of individual losses is the sum of the individual losses for a Participant $\mathrm{C}$ resulting from all products that are exchanged.

Note: if all six Participants B purchase a product with the smallest possible individual loss (0) for Participants C or do not purchase a product, Participants C's payment is 100; if all six Participants B purchase a product with the largest possible individual loss (10) for each Participant C, Participant C's payment is $\mathbf{4 0 .}$

\section{To summarize: Part II is identical to Part I.}

Do you have any further questions? If yes, please raise your hand. We will come to you at your workplace. Otherwise, we ask you to click the "OK” button. 


\section{Instructions for Part II}

\section{$\underline{\text { A. THE MARKET GAME }}$}

As previously announced, the market activity remains the same in Part II.

- Participants A are sellers. At the beginning of each period, each Participant A makes an offer to sell a product. To do so, he has to determine the price and the type (i.e. the total loss for Participant C) of the product he would like to offer.

Participants B are buyers. Each Participant B can then choose to buy one product from one of the Participants A, or can choose not to buy a product.

Participants C can neither sell nor buy, but they can incur losses due to the transactions between Participants A and B.

Your role in Part II is the same as it was in Part I.

\section{B. PAYMENTS OF PARTICIPANTS}

In Part II, the way payments are determined is different than in Part I.

In each period, each Participant A and each Participant C initially receives an endowment of 100 points as in Part I. But the endowments of the Participants B now change. Specifically, each Participant B now receives an initial endowment of 200 points in each period.

The payments of Participant A (seller), Participant B (buyer), and Participant C in a period are thus determined as follows: 
- Participant A's payment:

$$
100+\text { quantity sold } x \text { (price - production cost) }
$$

where the production cost, between 20 and 30, depends on the type of product offered, as shown in Table 1.

Note: if no Participant B accepts Participant A’s sales offer, Participant A’s payment is 100.

Participant B's payment:

- If Participant B makes a purchase:

$$
200+70-\text { price }
$$

- If Participant B does not make a purchase, his payment is 200.

- Participant C’s payment:

\section{0 - sum of individual losses}

where the sum of individual losses is the sum of the individual losses for a Participant $\mathrm{C}$ resulting from all products that are exchanged.

Note: if all six Participants B purchase a product with the smallest possible individual loss (0) for Participants C or do not purchase a product, Participants C's payment is 100; if all six Participants B purchase a product with the largest possible individual loss (10) for each Participant C, Participant C’s payment is $\mathbf{4 0 .}$

To summarize: Part II is identical to Part I except that the initial endowments of Participants $B$ are higher.

Do you have any further questions? If yes, please raise your hand. We will come to you at your workplace. Otherwise, we ask you to click the "OK” button. 


\section{Instructions for Part II}

\section{$\underline{\text { A. THE MARKET GAME }}$}

As previously announced, the market activity remains the same in Part II.

- Participants A are sellers. At the beginning of each period, each Participant A makes an offer to sell a product. To do so, he has to determine the price and the type (i.e. the total loss for Participant C) of the product he would like to offer.

Participants B are buyers. Each Participant B can then choose to buy one product from one of the Participants A, or can choose not to buy a product.

Participants C can neither sell nor buy, but they can incur losses due to the transactions between Participants A and B.

Your role in Part II is the same as it was in Part I.

\section{B. PAYMENTS OF PARTICIPANTS}

In Part II, the way payments are determined is different than in Part I.

In each period, each Participant A and each Participant C initially receives an endowment of 100 points as in Part I. But the endowments of the Participants B now change. Specifically, each Participant B now receives an initial endowment of 400 points in each period.

The payments of Participant A (seller), Participant B (buyer), and Participant C in a period are thus determined as follows: 
- Participant A's payment:

$$
100+\text { quantity sold } x \text { (price - production cost) }
$$

where the production cost, between 20 and 30, depends on the type of product offered, as shown in Table 1.

Note: if no Participant B accepts Participant A’s sales offer, Participant A’s payment is 100.

Participant B's payment:

- If Participant B makes a purchase:

$$
400+70-\text { price }
$$

- If Participant B does not make a purchase, his payment is $\mathbf{4 0 0 .}$

- Participant C’s payment:

\section{0 - sum of individual losses}

where the sum of individual losses is the sum of the individual losses for a Participant $\mathrm{C}$ resulting from all products that are exchanged.

Note: if all six Participants B purchase a product with the smallest possible individual loss (0) for Participants C or do not purchase a product, Participants C's payment is 100; if all six Participants B purchase a product with the largest possible individual loss (10) for each Participant C, Participant C’s payment is $\mathbf{4 0 .}$

To summarize: Part II is identical to Part I except that the initial endowments of Participants $B$ are higher.

Do you have any further questions? If yes, please raise your hand. We will come to you at your workplace. Otherwise, we ask you to click the "OK” button. 


\section{Instructions for Part II}

\section{A. THE MARKET GAME}

As previously announced, the market activity remains the same in Part II.

- Participants A are sellers. At the beginning of each period, each Participant A makes an offer to sell a product. To do so, he has to determine the price and the type (i.e. the total loss for Participant C) of the product he would like to offer.

Participants B are buyers. Each Participant B can then choose to buy one product from one of the Participants A, or can choose not to buy a product.

Participants C can neither sell nor buy, but they can incur losses due to the transactions between Participants A and B.

Your role in Part II is the same as it was in Part I.

\section{B. PAYMENTS OF PARTICIPANTS}

In Part II, the way payments are determined is different than in Part I.

In each period, each Participant $A$ and each Participant $C$ initially receives an endowment of 100 points as in Part I. But the endowments of some Participants B now change. Specifically, four Participants B now each receive an initial endowment of 100 points and two Participants $B$ now each receive an initial endowment of 400 points. The endowment that a particular Participant B receives will be randomly determined and will be the same for all of Part II. Each Participant B will be informed about the amount of their initial endowment on their screen once Part II begins.

The payments of Participant A (seller), Participant B (buyer), and Participant C in a period are thus determined as follows: 
- Participant A's payment:

$$
100+\text { quantity sold } x \text { (price - production cost) }
$$

where the production cost, between 20 and 30, depends on the type of product offered, as shown in Table 1.

Note: if no Participant B accepts Participant A’s sales offer, Participant A’s payment is 100.

- Participant B’s payment:

If Participant B initially receives 100:

- If Participant B makes a purchase:

$$
100+70-\text { price }
$$

- If Participant B does not make a purchase, his payment is $\mathbf{1 0 0 .}$

If Participant B initially receives 400:

- If Participant B makes a purchase:

$$
400+70-\text { price }
$$

- If Participant B does not make a purchase, his payment is $\mathbf{4 0 0 .}$

- Participant C's payment:

$$
100 \text { - sum of individual losses }
$$

where the sum of individual losses is the sum of the individual losses for a Participant C resulting from all products that are exchanged.

Note: if all six Participants B purchase a product with the smallest possible individual loss (0) for Participants C or do not purchase a product, Participants C's payment is 100; if all six Participants B purchase a product with the largest possible individual loss (10) for each Participant C, Participant C’s payment is 40.

To summarize: Part II is identical to Part I except that the initial endowments of some Participants B are higher.

Do you have any further questions? If yes, please raise your hand. We will come to you at your workplace. Otherwise, we ask you to click the "OK” button. 